\title{
On the origins of approximations for stochastic chemical kinetics
}

\author{
Eric L. Haseltine \\ Division of Chemistry and Chemical Engineering 210-41, California Institute of Technology, \\ Pasadena, California 91125
}

\author{
James B. Rawlings ${ }^{a)}$ \\ Department of Chemical and Biological Engineering, University of Wisconsin-Madison, \\ Madison, Wisconsin 53706-1607
}

(Received 20 June 2005; accepted 18 August 2005; published online 27 October 2005)

\begin{abstract}
This paper considers the derivation of approximations for stochastic chemical kinetics governed by the discrete master equation. Here, the concepts of (1) partitioning on the basis of fast and slow reactions as opposed to fast and slow species and (2) conditional probability densities are used to derive approximate, partitioned master equations, which are Markovian in nature, from the original master equation. Under different conditions dictated by relaxation time arguments, such approximations give rise to both the equilibrium and hybrid (deterministic or Langevin equations coupled with discrete stochastic simulation) approximations previously reported. In addition, the derivation points out several weaknesses in previous justifications of both the hybrid and equilibrium systems and demonstrates the connection between the original and approximate master equations. Two simple examples illustrate situations in which these two approximate methods are applicable and demonstrate the two methods' efficiencies. (C) 2005 American Institute of Physics.
\end{abstract}

[DOI: 10.1063/1.2062048]

\section{INTRODUCTION}

Exact methods are available for the simulation of isothermal, well-mixed stochastic chemical kinetics. ${ }^{1-4}$ As increasingly complex physical systems are modeled, however, these methods become difficult to solve because the computational burden scales with the number of reaction events. ${ }^{3}$ Consequently, recent efforts have focused on approximations to reduce this computational burden. These efforts consist of approximating either the entire master equation (e.g., the $\tau$-leaping ${ }^{5-7}$ and binomial-leaping ${ }^{8,9}$ methods) or a portion of the master equation (the so-called partitioning methods). In this paper, we focus our attention on the partitioning methods and their derivations.

The master equation has been partitioned using both the species number and the extent of reaction coordinates. We first review contributions that partition on the basis of the species number coordinate. To the best of our knowledge, Janssen $^{10,11}$ and Vlad and Pop ${ }^{12}$ are the first to examine the adiabatic elimination of fast-relaxing variables in discrete stochastic chemical kinetics using this coordinate. These works draw inspiration from the early contributions of both Haken ${ }^{13}$ and van Kampen. ${ }^{14}$ In fact, Haken outlines a general procedure for eliminating fast-relaxing variables from the discrete master equations. ${ }^{13}$ This general derivation nominally encompasses any coordinate system, and as we demonstrate later in this paper the proper choice of coordinate system can lead to both convenient reductions in the structure of the master equation and justification of the adiabatic hypothesis. Rao and Arkin use the quasi-steady-state assumption to approximate the fast species and propose algorithms for nu-

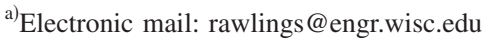

merically simulating the resulting reduced system. ${ }^{15}$ Cao et $a l .{ }^{16}$ equilibrate the fast species using an equilibrium approximation on a virtual fast subsystem, and then derive a slow-scale stochastic simulation algorithm for the slow species.

The extent of the reaction coordinate system has been used by several authors to partition the master equation. Haseltine and Rawlings ${ }^{17}$ partition chemical reactions into fast and slow reactions using an irreversible extent coordinate, approximate the extents corresponding to the fast reactions either using Langevin or deterministic equations, and propose several algorithms to simulate the resulting system. Here, the authors postulated the form of the reduced master equation. In contrast to this approach, both Haseltine ${ }^{18}$ and Goutsias ${ }^{19}$ partition chemical reactions into fast and slow reactions again using an irreversible extent coordinate ${ }^{20}$ but derive the form of the reduced master equation using orderof-magnitude arguments. By making various approximations on the fast extents, these authors then derive stochastic simulation algorithms for the slow extents.

In this paper, we use the constructs of (1) partitioning on the basis of fast and slow reactions using a net extent coordinate and (2) conditional probability densities to derive approximate, partitioned master equations, which are Markovian in nature, from the original master equation. We demonstrate that under different conditions dictated by the characteristic relaxation times, such approximations give rise to both the hybrid approximation previously presented by Haseltine and Rawlings ${ }^{17}$ and the equilibrium approximation previously reported by Cao et al. ${ }^{16}$ and Goutsias. ${ }^{19}$ Our derivation points out several weaknesses in our previous justification of the hybrid system ${ }^{17}$ and, in our opinion, presents an alternative and simpler method for deriving the equilibrium 
approximation than those proposed by either Cao et al. ${ }^{16}$ or Goutsias. ${ }^{19}$ Additionally, the derivation demonstrates the connections between the original and approximate master equations. Two simple examples illustrate situations in which these methods are applicable.

\section{STOCHASTIC PARTITIONING}

For chemical kinetics, the master equation is usually presented using the species number coordinate. An alternative coordinate is the extent of reaction, and working in this coordinate can prove useful as demonstrated by Vlad and Ross $^{21,22}$ and Ishida. ${ }^{23}$ Here, the key ideas are to (1) model the state of the reacting system using the net extents of the reactions as opposed to the numbers of molecules of each species and (2) partition the state into subsets of "fast" and "slow" extents. With these modeling choices we can exploit the structure of the chemical master equation, the governing equation for the evolution of the system probability density, by making order-of-magnitude arguments. We then derive the master equations that govern the fast-and slow-extent subsets. This section presents these steps in greater detail.

We model the state of the system, $\mathbf{x}$, using a net reaction extent for each reaction. Note that reversible reactions are modeled using only one net extent. This choice deviates from previous derivations, ${ }^{17-19}$ which model reversible reactions as two extents (one for the forward reaction and one for the reverse reaction), and deserves some additional attention. Consider the simple example

$$
A \rightleftharpoons B
$$

with initial condition of ten molecules of $A$ and zero $B$. If we choose to model this system using a single net extent, then there are a finite number of values (11) that this extent may take. On the other hand, if we model this system using two extents (one for each of the forward and reverse reactions), then there are an infinite number of values that the extent might take because we require only that the sum of the two extents yield non-negative numbers of molecules for both species. Thus, under standard assumptions such as conservation of elements, a finite number of reactions, and finite stoichiometries for reaction events, the net extent representation yields a master equation with a finite number of entries, whereas the irreversible extent representation yields an infinite number of entries. To facilitate the analysis of the master equation, we have chosen to use the net extent representation.

In this representation, species numbers are, of course, related to the reaction extents by

$$
\mathbf{n}=\mathbf{n}_{0}+\boldsymbol{\nu}^{T} \mathbf{x}
$$

and the choice of the reaction extent coordinates is motivated only by improved clarity when treating large and small reaction propensities in the subsequent development. Assuming $m$ net reaction extents among $p$ chemical species, we have the following:
- $\mathbf{x}$ is the state of the system in terms of net reaction extents ( an $m$-vector),

- $\mathbf{n}$ is the number of molecules of each species (a $p$-vector),

- $\mathbf{n}_{0}$ is the initial number of molecules (a $p$-vector), and

- $\boldsymbol{\nu}$ is the stoichiometric matrix (an $m \times p$-matrix).

The upper and lower bounds of $\mathbf{x}$ are constrained by the limiting reactant species. We arbitrarily set the initial condition to zero. Given assumptions outlined by Gillespie, ${ }^{24}$ the governing equation for this system is the chemical master equation

$$
\begin{aligned}
\frac{d P(\mathbf{x} ; t)}{d t}= & \sum_{k=1}^{m} a_{k}^{f}\left(\mathbf{x}-\boldsymbol{I}_{k}\right) P\left(\mathbf{x}-\boldsymbol{I}_{k} ; t\right) \\
& +a_{k}^{r}\left(\mathbf{x}+\boldsymbol{I}_{k}\right) P\left(\mathbf{x}+\boldsymbol{I}_{k} ; t\right)-\left(a_{k}^{f}(\mathbf{x})+a_{k}^{r}(\mathbf{x})\right) P(\mathbf{x} ; t)
\end{aligned}
$$

in which

- $P(\mathbf{x} ; t)$ is the probability that the system is in state $\mathbf{x}$ at time $t$,

- $a_{k}^{f}(\mathbf{x}) d t$ is the probability to order $d t$ that the $k$ th forward reaction occurs in the time interval $[t, t+d t)$,

- $a_{k}^{r}(\mathbf{x}) d t$ is the probability to order $d t$ that the $k$ th reverse reaction occurs in the time interval $[t, t+d t)$, and

- $\boldsymbol{I}_{k}$ is the $k$ th column of the $(m \times m)$-identity matrix $\boldsymbol{I}$.

Here, each net extent is characterized by a forward and reverse reaction with propensities $a_{k}^{f}(\mathbf{x})$ and $a_{k}^{r}(\mathbf{x})$, respectively. Potentially either or both of these propensities may be zero for a given $\mathbf{x}$. The structure of $\boldsymbol{I}$ arises for this particular chemical master equation because of the net extent coordinate system. Also, we have implicitly conditioned the master equation (2) on a specific initial condition, i.e., $\mathbf{n}_{0}$. Generalizing the analysis presented in this paper to a distribution of initial conditions $\left(\mathbf{n}_{0,1}, \ldots, \mathbf{n}_{0, n}\right)$ is straightforward due to the relation

$$
P\left(\mathbf{x} \mid \mathbf{n}_{0,1}, \ldots, \mathbf{n}_{0, n} ; t\right)=\sum_{j} P\left(\mathbf{x} \mid \mathbf{n}_{0, j} ; t\right) P\left(\mathbf{n}_{0, j}\right)
$$

and the fact that the values of $P\left(\mathbf{n}_{0, j}\right)$ are specified in the initial condition.

Now we partition the extents into two subsets: those that have small net propensity functions $\left(\left|a_{k}^{f}(\mathbf{x})+a_{k}^{r}(\mathbf{x})\right|\right.$ 's $)$, and those that have large propensity functions. We designate these subsets of $\mathbf{x}$ as the $(m-l)$-vector $\mathbf{y}$ and the $l$-vector $\mathbf{z}$, respectively. Note that 


$$
\mathbf{x}=\left[\begin{array}{l}
\mathbf{y} \\
\mathbf{z}
\end{array}\right] \begin{gathered}
\text { slow extents } \\
\text { fast extents }
\end{gathered}, \quad \boldsymbol{I}=\left[\begin{array}{cc}
\boldsymbol{I}^{y} & 0 \\
0 & \boldsymbol{I}^{z}
\end{array}\right]
$$

in which $\boldsymbol{I}^{y}$ and $\boldsymbol{I}^{z}$ are $(m-l \times m-l)$ - and $(l \times l)$-identity matrices, respectively. Denoting the total extent space as the set $\mathbb{X}$, we define a subset $\mathbb{X}_{p} \subset \mathbb{X}$ for which

$$
\begin{aligned}
& \left|c_{k}^{f}(\mathbf{y}, \mathbf{z})+c_{k}^{r}(\mathbf{y}, \mathbf{z})\right| \gg\left|b_{j}^{f}(\mathbf{y}, \mathbf{z})+b_{j}^{r}(\mathbf{y}, \mathbf{z})\right| \\
& \forall 1 \leqslant k \leqslant l, \quad 1 \leqslant j \leqslant m-l, \quad\left[\begin{array}{l}
\mathbf{y} \\
\mathbf{z}
\end{array}\right] \in \mathbb{X}_{p}
\end{aligned}
$$

in which we have partitioned the reaction propensities into groups of fast $\left(c_{j}^{f}\right.$ and $\left.c_{j}^{r}\right)$ and slow $\left(b_{j}^{f}\right.$ and $\left.b_{j}^{r}\right)$

$$
\left[\begin{array}{c}
a_{1}^{f}(\mathbf{y}, \mathbf{z} ; t) \\
\vdots \\
a_{m-l}^{f}(\mathbf{y}, \mathbf{z} ; t) \\
a_{1}^{r}(\mathbf{y}, \mathbf{z} ; t) \\
\vdots \\
a_{m-l}^{r}(\mathbf{y}, \mathbf{z} ; t)
\end{array}\right]=\left[\begin{array}{c}
b_{1}^{f}(\mathbf{y}, \mathbf{z} ; t) \\
\vdots \\
b_{m-l}^{f}(\mathbf{y}, \mathbf{z} ; t) \\
b_{1}^{r}(\mathbf{y}, \mathbf{z} ; t) \\
\vdots \\
b_{m-l}^{r}(\mathbf{y}, \mathbf{z} ; t)
\end{array}\right] \text { slow reaction },
$$

$$
\left[\begin{array}{c}
a_{m-l+1}^{f}(\mathbf{y}, \mathbf{z} ; t) \\
\vdots \\
a_{m}^{f}(\mathbf{y}, \mathbf{z} ; t) \\
a_{m-l+1}^{r}(\mathbf{y}, \mathbf{z} ; t) \\
\vdots \\
a_{m}^{r}(\mathbf{y}, \mathbf{z} ; t)
\end{array}\right]=\left[\begin{array}{c}
c_{1}^{f}(\mathbf{y}, \mathbf{z} ; t) \\
\vdots \\
c_{l}^{f}(\mathbf{y}, \mathbf{z} ; t) \\
c_{1}^{r}(\mathbf{y}, \mathbf{z} ; t) \\
\vdots \\
c_{l}^{r}(\mathbf{y}, \mathbf{z} ; t)
\end{array}\right] \text { fast reaction }
$$

We also define the sets $\mathbb{Y}$ and $\mathbb{Z}$ to span all possible combinations of slow and fast reaction extents, respectively. $\mathrm{Y}_{p} \subset \mathbb{Y}$ and $\mathbb{Z}_{p} \subset \mathbb{Z}$ are the slow and fast reaction extents corresponding to Eq. (5).

Thus, Eq. (2) becomes

$$
\begin{aligned}
\frac{d P(\mathbf{y}, \mathbf{z} ; t)}{d t}= & \sum_{j=1}^{m-l} b_{j}^{f}\left(\mathbf{y}-\boldsymbol{I}_{j}^{y}, \mathbf{z}\right) P\left(\mathbf{y}-\boldsymbol{I}_{j}^{y}, \mathbf{z} ; t\right) \\
& +b_{j}^{r}\left(\mathbf{y}+\boldsymbol{I}_{j}^{y}, \mathbf{z}\right) P\left(\mathbf{y}+\boldsymbol{I}_{j}^{y}, \mathbf{z} ; t\right) \\
& -\left(b_{j}^{f}(\mathbf{y}, \mathbf{z})+b_{j}^{r}(\mathbf{y}, \mathbf{z})\right) P(\mathbf{y}, \mathbf{z} ; t) \\
& +\sum_{k=1}^{l} c_{k}^{f}\left(\mathbf{y}, \mathbf{z}-\boldsymbol{I}_{k}^{z}\right) P\left(\mathbf{y}, \mathbf{z}-\boldsymbol{I}_{k}^{z} ; t\right) \\
& +c_{k}^{r}\left(\mathbf{y}, \mathbf{z}+\boldsymbol{I}_{k}^{z}\right) P\left(\mathbf{y}, \mathbf{z}+\boldsymbol{I}_{k}^{z} ; t\right) \\
& -\left(c_{k}^{f}(\mathbf{y}, \mathbf{z})+c_{k}^{r}(\mathbf{y}, \mathbf{z})\right) P(\mathbf{y}, \mathbf{z} ; t) .
\end{aligned}
$$

Ultimately, we are interested in determining an approximate governing equation for the evolution of the joint density, $P(\mathbf{y}, \mathbf{z} ; t)$, in regimes where fast-reaction propensities are much greater than slow-reaction propensities.

By defining the conditional and marginal probabilities over this subset as

$$
\begin{aligned}
& P(\mathbf{y}, \mathbf{z} ; t)=P(\mathbf{z} \mid \mathbf{y} ; t) P(\mathbf{y} ; t) \quad \forall \mathbf{y} \in \mathbb{Y}_{p}, \quad \mathbf{z} \in \mathbb{Z}_{p}, \\
& P(\mathbf{y} ; t)=\sum_{\mathbf{z} \in Z_{p}} P(\mathbf{y}, \mathbf{z} ; t) \quad \forall \mathbf{y} \in \mathbb{Y}_{p},
\end{aligned}
$$

we can alternatively derive evolution equations for both the marginal probability of the slow reactions, $P(\mathbf{y} ; t)$, and the probability of the fast reactions conditioned on the slow reactions, $P(\mathbf{z} \mid \mathbf{y} ; t)$. Consequently, we then know how the fast and slow reactions evolve. Also, this partitioning is similar to that used by Rao and Arkin, ${ }^{15}$ who partition the master equation by species to treat the quasi-steady-state assumption. We partition by reaction extents to treat fast and slow reactions.

\section{A. Slow-reaction subset}

We first address the subset of slow-reaction extents $\mathbf{y}$. From the definition of the marginal density,

$$
P(\mathbf{y} ; t)=\sum_{\mathbf{z} \in Z_{p}} P(\mathbf{y}, \mathbf{z} ; t) \quad \forall \mathbf{y} \in \mathbb{Y}_{p} .
$$

Differentiating Eq. (11) with respect to time yields

$$
\frac{d P(\mathbf{y} ; t)}{d t}=\sum_{\mathbf{z} \in \mathbb{Z}_{p}} \frac{d P(\mathbf{y}, \mathbf{z} ; t)}{d t} \quad \forall \mathbf{y} \in \mathbb{Y}_{p} .
$$

Now substitute the master equation (8) into Eq. (12) and manipulate to yield

$$
\begin{aligned}
\frac{d P(\mathbf{y} ; t)}{d t}= & \sum_{\mathbf{z} \in Z_{p}}\left(\sum_{j=1}^{m-l} b_{j}^{f}\left(\mathbf{y}-\boldsymbol{I}_{j}^{y}, \mathbf{z}\right) P\left(\mathbf{y}-\boldsymbol{I}_{j}^{y}, \mathbf{z} ; t\right)+b_{j}^{r}\left(\mathbf{y}+\boldsymbol{I}_{j}^{y}, \mathbf{z}\right) P\left(\mathbf{y}+\boldsymbol{I}_{j}^{y}, \mathbf{z} ; t\right)-\left(b_{j}^{f}(\mathbf{y}, \mathbf{z})+b_{j}^{r}(\mathbf{y}, \mathbf{z})\right) P(\mathbf{y}, \mathbf{z} ; t)\right. \\
& \left.+\sum_{k=1}^{l} c_{k}^{f}\left(\mathbf{y}, \mathbf{z}-\boldsymbol{I}_{k}^{z}\right) P\left(\mathbf{y}, \mathbf{z}-\boldsymbol{I}_{k}^{z} ; t\right)+c_{k}^{r}\left(\mathbf{y}, \mathbf{z}+\boldsymbol{I}_{k}^{z}\right) P\left(\mathbf{y}, \mathbf{z}+\boldsymbol{I}_{k}^{z} ; t\right)-\left(c_{k}^{f}(\mathbf{y}, \mathbf{z})+c_{k}^{r}(\mathbf{y}, \mathbf{z})\right) P(\mathbf{y}, \mathbf{z} ; t)\right) \quad \forall \mathbf{y} \in \mathbb{Y}_{p}
\end{aligned}
$$




$$
\begin{aligned}
& =\sum_{j=1}^{m-l} \sum_{\mathbf{z} \in Z_{p}} b_{j}^{f}\left(\mathbf{y}-\boldsymbol{I}_{j}^{y}, \mathbf{z}\right) P\left(\mathbf{y}-\boldsymbol{I}_{j}^{y}, \mathbf{z} ; t\right)+b_{j}^{r}\left(\mathbf{y}+\boldsymbol{I}_{j}^{y}, \mathbf{z}\right) P\left(\mathbf{y}+\boldsymbol{I}_{j}^{y}, \mathbf{z} ; t\right)-\left(b_{j}^{f}(\mathbf{y}, \mathbf{z})+b_{j}^{r}(\mathbf{y}, \mathbf{z})\right) P(\mathbf{y}, \mathbf{z} ; t) \\
& +\sum_{k=1}^{l} \sum_{\mathbf{z} \in Z_{p}} c_{k}^{f}\left(\mathbf{y}, \mathbf{z}-\boldsymbol{I}_{k}^{z}\right) P\left(\mathbf{y}, \mathbf{z}-\boldsymbol{I}_{k}^{z} ; t\right)+c_{k}^{r}\left(\mathbf{y}, \mathbf{z}+\boldsymbol{I}_{k}^{z}\right) P\left(\mathbf{y}, \mathbf{z}+\boldsymbol{I}_{k}^{z} ; t\right)-\left(c_{k}^{f}(\mathbf{y}, \mathbf{z})+c_{k}^{r}(\mathbf{y}, \mathbf{z})\right) P(\mathbf{y}, \mathbf{z} ; t) \quad \forall \mathbf{y} \in \mathbb{Y}_{p} \\
& m-l \\
= & \sum_{j=1} \sum_{\mathbf{z} \in \mathbb{Z}_{p}} b_{j}^{f}\left(\mathbf{y}-\boldsymbol{I}_{j}^{y}, \mathbf{z}\right) P\left(\mathbf{y}-\boldsymbol{I}_{j}^{y}, \mathbf{z} ; t\right)+b_{j}^{r}\left(\mathbf{y}+\boldsymbol{I}_{j}^{y}, \mathbf{z}\right) P\left(\mathbf{y}+\boldsymbol{I}_{j}^{y}, \mathbf{z} ; t\right)-\left(b_{j}^{f}(\mathbf{y}, \mathbf{z})+b_{j}^{r}(\mathbf{y}, \mathbf{z})\right) P(\mathbf{y}, \mathbf{z} ; t) \quad \forall \mathbf{y} \in \mathbb{Y}_{p} .
\end{aligned}
$$

If $\mathbb{Z}_{p}=\mathbb{Z}$, then Eq. (15) is exact; otherwise, we obtain a boundary condition equating the probability fluxes between the partitions [i.e., $P(\mathbf{y}) \forall \mathbf{y} \in \mathbb{Y}_{p}$ and $P(\mathbf{y}) \forall \mathbf{y} \notin \mathbb{Y}_{p}$ ]. Also, if we rewrite the joint density in terms of the conditional density using the definition

$$
P(\mathbf{y}, \mathbf{z} ; t)=P(\mathbf{z} \mid \mathbf{y} ; t) P(\mathbf{y} ; t) \quad \forall \mathbf{y} \in \mathbb{Y}_{p}, \quad \mathbf{z} \in \mathbb{Z}_{p},
$$

then one interpretation of this analysis is that the evolution of the marginal $P(\mathbf{y} ; t)$ depends on the conditional density $P(\mathbf{z} \mid \mathbf{y} ; t)$. We consider deriving an evolution equation for the conditional density $P(\mathbf{z} \mid \mathbf{y} ; t)$ next.

\section{B. Fast-reaction subset}

We now address the evolution of the probability density for the subset of fast reactions conditioned on the subset of slow reactions, $P(\mathbf{z} \mid \mathbf{y} ; t)$. For our starting point, we use order-of-magnitude arguments, i.e., Eq. (5), to approximate the original master equation (8) as

$$
\begin{aligned}
\frac{d P(\mathbf{y}, \mathbf{z} ; t)}{d t} \approx & \sum_{k=1}^{l} c_{k}^{f}\left(\mathbf{y}, \mathbf{z}-\boldsymbol{I}_{k}^{z}\right) P\left(\mathbf{y}, \mathbf{z}-\boldsymbol{I}_{k}^{z} ; t\right) \\
& +c_{k}^{r}\left(\mathbf{y}, \mathbf{z}+\boldsymbol{I}_{k}^{z}\right) P\left(\mathbf{y}, \mathbf{z}+\boldsymbol{I}_{k}^{z} ; t\right) \\
& -\left(c_{k}^{f}(\mathbf{y}, \mathbf{z})+c_{k}^{r}(\mathbf{y}, \mathbf{z})\right) P(\mathbf{y}, \mathbf{z} ; t) \\
& \forall \mathbf{y} \in \mathbb{Y}_{p}, \quad \mathbf{z} \in \mathbb{Z}_{p} .
\end{aligned}
$$

We define this approximate joint density as $P_{A}(\mathbf{y}, \mathbf{z} ; t)$, and thus its evolution equation is

$$
\begin{aligned}
\frac{d P_{A}(\mathbf{y}, \mathbf{z} ; t)}{d t} \triangleq & \sum_{k=1}^{l} c_{k}^{f}\left(\mathbf{y}, \mathbf{z}-\boldsymbol{I}_{k}^{z}\right) P_{A}\left(\mathbf{y}, \mathbf{z}-\boldsymbol{I}_{k}^{z} ; t\right) \\
& +c_{k}^{r}\left(\mathbf{y}, \mathbf{z}+\boldsymbol{I}_{k}^{z}\right) P_{A}\left(\mathbf{y}, \mathbf{z}+\boldsymbol{I}_{k}^{z} ; t\right) \\
& -\left(c_{k}^{f}(\mathbf{y}, \mathbf{z})+c_{k}^{r}(\mathbf{y}, \mathbf{z})\right) P_{A}(\mathbf{y}, \mathbf{z} ; t) \\
& \forall \mathbf{y} \in \mathbb{Y}_{p}, \quad \mathbf{z} \in \mathbb{Z}_{p} .
\end{aligned}
$$

Following Rao and Arkin, ${ }^{15}$ we define the joint density $P_{A}(\mathbf{y}, \mathbf{z} ; t)$ as the product of the desired conditional density $P_{A}(\mathbf{z} \mid \mathbf{y} ; t)$ and the marginal density $P_{A}(\mathbf{y} ; t)$ :

$$
P_{A}(\mathbf{y}, \mathbf{z} ; t)=P_{A}(\mathbf{z} \mid \mathbf{y} ; t) P_{A}(\mathbf{y} ; t) \quad \forall \mathbf{y} \in \mathbb{Y}_{p}, \quad \mathbf{z} \in \mathbb{Z}_{p} .
$$

Differentiating Eq. (19) with respect to time yields

$$
\begin{gathered}
\frac{d P_{A}(\mathbf{y}, \mathbf{z} ; t)}{d t}=\frac{d P_{A}(\mathbf{z} \mid \mathbf{y} ; t)}{d t} P_{A}(\mathbf{y} ; t)+\frac{d P_{A}(\mathbf{y} ; t)}{d t} P_{A}(\mathbf{z} \mid \mathbf{y} ; t) \\
\forall \mathbf{y} \in \mathbb{Y}_{p}, \quad \mathbf{z} \in \mathbb{Z}_{p} .
\end{gathered}
$$

Solving Eq. (20) for the desired conditional derivative yields

$$
\begin{aligned}
\frac{d P_{A}(\mathbf{z} \mid \mathbf{y} ; t)}{d t}= & \frac{1}{P_{A}(\mathbf{y} ; t)}\left(\frac{d P_{A}(\mathbf{y}, \mathbf{z} ; t)}{d t}\right. \\
& \left.-\frac{d P_{A}(\mathbf{y} ; t)}{d t} P_{A}(\mathbf{z} \mid \mathbf{y} ; t)\right) \\
& \forall \mathbf{y} \in \mathbb{Y}_{p}, \quad \mathbf{z} \in \mathbb{Z}_{p} .
\end{aligned}
$$

Equation (21) obviously requires the probability $P_{A}(\mathbf{y} ; t)$ to be nonzero for $t_{0}<t<\infty$. Under standard assumptions such as conservation of elements, a finite number of reactions, and finite stoichiometries for reaction events, it can be shown that this statement is true for the net extent coordinate. Evaluating the marginal evolution equation by summing Eq. (18) over the fast extents $\mathbf{z}$ yields

$$
\begin{aligned}
\frac{d P_{A}(\mathbf{y} ; t)}{d t}= & \sum_{\mathbf{z} \in Z_{p}} \sum_{k=1}^{l} c_{k}^{f}\left(\mathbf{y}, \mathbf{z}-\boldsymbol{I}_{k}^{z}\right) P_{A}\left(\mathbf{y}, \mathbf{z}-\boldsymbol{I}_{k}^{z} ; t\right) \\
& +c_{k}^{r}\left(\mathbf{y}, \mathbf{z}+\boldsymbol{I}_{k}^{z}\right) P_{A}\left(\mathbf{y}, \mathbf{z}+\boldsymbol{I}_{k}^{z} ; t\right) \\
& -\left(c_{k}^{f}(\mathbf{y}, \mathbf{z})+c_{k}^{r}(\mathbf{y}, \mathbf{z})\right) P_{A}(\mathbf{y}, \mathbf{z} ; t) \\
& \forall \mathbf{y} \in \mathbb{Y}_{p}
\end{aligned}
$$

$$
=0 \quad \forall \mathbf{y} \in \mathbb{Y}_{p} \text {. }
$$

Consequently, Eq. (21) becomes 


$$
\begin{aligned}
\frac{d P_{A}(\mathbf{z} \mid \mathbf{y} ; t)}{d t}= & \left(\sum_{k=1}^{l} c_{k}^{f}\left(\mathbf{y}, \mathbf{z}-\boldsymbol{I}_{k}^{z}\right) P_{A}\left(\mathbf{y}, \mathbf{z}-\boldsymbol{I}_{k}^{z} ; t\right)\right. \\
& +c_{k}^{r}\left(\mathbf{y}, \mathbf{z}+\boldsymbol{I}_{k}^{z}\right) P_{A}\left(\mathbf{y}, \mathbf{z}+\boldsymbol{I}_{k}^{z} ; t\right) \\
& \left.-\left(c_{k}^{f}(\mathbf{y}, \mathbf{z})+c_{k}^{r}(\mathbf{y}, \mathbf{z})\right) P_{A}(\mathbf{y}, \mathbf{z} ; t)\right) \frac{1}{P_{A}(\mathbf{y} ; t)} \\
\forall & \mathbf{y} \in \mathbb{Y}_{p}, \quad \mathbf{z} \in \mathbb{Z}_{p} \\
& \sum_{k=1}^{l} c_{k}^{f}\left(\mathbf{y}, \mathbf{z}-\boldsymbol{I}_{k}^{z}\right) P_{A}\left(\mathbf{z}-\boldsymbol{I}_{k}^{z} \mid \mathbf{y} ; t\right) \\
& +c_{k}^{r}\left(\mathbf{y}, \mathbf{z}+\boldsymbol{I}_{k}^{z}\right) P_{A}\left(\mathbf{z}+\boldsymbol{I}_{k}^{z} \mid \mathbf{y} ; t\right) \\
& -\left(c_{k}^{f}(\mathbf{y}, \mathbf{z})+c_{k}^{r}(\mathbf{y}, \mathbf{z})\right) P_{A}(\mathbf{z} \mid \mathbf{y} ; t) \\
\forall & \mathbf{y} \in \mathbb{Y} p, \quad \mathbf{z} \in \mathbb{Z}_{p},
\end{aligned}
$$

which is the desired closed-form expression for the conditional density $P_{A}(\mathbf{z} \mid \mathbf{y} ; t)$.

\section{The combined system}

We approximate the joint density $P(\mathbf{y}, \mathbf{z} ; t)$ as

$$
P(\mathbf{y}, \mathbf{z} ; t) \approx P_{A}(\mathbf{z} \mid \mathbf{y} ; t) P(\mathbf{y} ; t) \quad \forall \mathbf{y} \in \mathbb{Y}_{p}, \quad \mathbf{z} \in \mathbb{Z}_{p} .
$$

Combining the evolution equations for the slow- and fastreaction extents, i.e., Eqs. (15) and (25), respectively, then yields the following coupled master equations:

$$
\begin{aligned}
\frac{d P(\mathbf{y} ; t)}{d t} \approx & \sum_{j=1}^{m-l}\left(\sum_{\mathbf{z} \in Z_{p}} b_{j}^{f}\left(\mathbf{y}-\boldsymbol{I}_{j}^{y}, \mathbf{z}\right) P_{A}\left(\mathbf{z} \mid \mathbf{y}-\boldsymbol{I}_{j}^{y} ; t\right)\right) P\left(\mathbf{y}-\boldsymbol{I}_{j}^{y} ; t\right) \\
+ & \left(\sum_{\mathbf{z} \in Z_{p}} b_{j}^{r}\left(\mathbf{y}+\boldsymbol{I}_{j}^{y}, \mathbf{z}\right) P_{A}\left(\mathbf{z} \mid \mathbf{y}+\boldsymbol{I}_{j}^{y} ; t\right)\right) P\left(\mathbf{y}+\boldsymbol{I}_{j}^{y} ; t\right) \\
& -\left(\sum_{\mathbf{z} \in Z_{p}}\left(b_{j}^{f}(\mathbf{y}, \mathbf{z})+b_{j}^{r}(\mathbf{y}, \mathbf{z})\right) P_{A}(\mathbf{z} \mid \mathbf{y} ; t)\right) P(\mathbf{y} ; t) \\
& \forall \mathbf{y} \in \mathbb{Y}_{p}, \\
\frac{d P_{A}(\mathbf{z} \mid \mathbf{y} ; t)}{d t}= & \sum_{k=1} c_{k}^{f}\left(\mathbf{y}, \mathbf{z}-\boldsymbol{I}_{k}^{z}\right) P_{A}\left(\mathbf{z}-\boldsymbol{I}_{k}^{z} \mid \mathbf{y} ; t\right) \\
& +c_{k}^{r}\left(\mathbf{y}, \mathbf{z}+\boldsymbol{I}_{k}^{z}\right) P_{A}\left(\mathbf{z}+\boldsymbol{I}_{k}^{z} \mid \mathbf{y} ; t\right) \\
& -\left(c_{k}^{f}(\mathbf{y}, \mathbf{z})+c_{k}^{r}(\mathbf{y}, \mathbf{z})\right) P_{A}(\mathbf{z} \mid \mathbf{y} ; t) \\
& \forall \mathbf{y} \in \mathbb{Y}_{p}, \quad \mathbf{z} \in \mathbb{Z}_{p}, \\
\frac{m}{d P(\mathbf{x} ; t)} & \sum_{k=1} a_{k}^{f}\left(\mathbf{x}-\boldsymbol{I}_{k}\right) P\left(\mathbf{x}-\boldsymbol{I}_{k} ; t\right) \\
+ & a_{k}^{r}\left(\mathbf{x}+\boldsymbol{I}_{k}\right) P\left(\mathbf{x}+\boldsymbol{I}_{k} ; t\right) \\
- & \left(a_{k}^{f}(\mathbf{x})+a_{k}^{r}(\mathbf{x})\right) P(\mathbf{x} ; t) \\
\forall & \mathbf{x} \notin \mathbb{X}_{p} .
\end{aligned}
$$

Additionally, the initial condition for the coupled master equations (27) can easily be derived from the initial condition for the original master equation (2). From these equa- tions, using order-of-magnitude arguments to partition the system has clearly had three effects. First, the original master equation given by Eq. (27c) still holds when the partitioning criteria are not met (i.e., $\mathbf{x} \notin \mathbb{X}_{p}$ ), whereas coupled, approximate equations govern the system evolution when the partitioning criteria are met (i.e., $\mathbf{x} \in \mathbb{X}_{p}$ ). Second, the coupled expressions for the marginal and conditional evolution equations in (27) are Markovian in nature. Finally, the approximate evolution equation for the fast extents conditioned on the slow extents, $P_{A}(\mathbf{z} \mid \mathbf{y})$, has completely decoupled from the slow extent marginal, $P(\mathbf{y})$. With these findings, we address the claim made by Cao et al. ${ }^{16}$ that the real fast system conditioned on the slow system is not Markovian. Indeed, density $P(\mathbf{z} \mid \mathbf{y} ; t)$ is not Markovian and solving for it would require solution of the joint density $P(\mathbf{y}, \mathbf{z} ; t)$. However, the approximate density $P_{A}(\mathbf{z} \mid \mathbf{y} ; t)$ calculated using order-ofmagnitude arguments is Markovian and independent of the slow marginal $P(\mathbf{y} ; t)$, which makes analysis tractable as is shown subsequently.

Exact solution of the coupled master equations (27) is at least as difficult as the original master equation (2) due to the fact that one must solve an individual master equation of the form of Eq. (27b) for every element of the slow conditional equation (27a). From a simulation perspective, Eq. (27) is also as difficult to evaluate as the original master equation (2) since both of the coupled master equations are discrete and time-varying. However, approximating the fast extents can significantly reduce the computational expense involved with simulating these coupled equations. Different approximations are applicable based on the characteristic relaxation times of the fast and slow extents. Next, we investigate two such approximations: an equilibrium approximation for the case in which the fast extents relax significantly faster than the slow extents, and a Langevin or deterministic approximation for the case in which both fast and slow extents relax at similar rates.

\section{The equilibrium approximation}

We first consider the case in which the relaxation time for the fast extents is significantly smaller than the expected time to the first slow reaction. To illustrate this case, we consider the simple example

$$
A \underset{k_{1}^{r}}{\stackrel{k_{1}^{f}}{\rightleftharpoons}} B \stackrel{k_{2}^{f}}{\longrightarrow} C .
$$

We denote the extents of reaction for this example as $\epsilon_{1}$ and $\epsilon_{2}$, and define the reaction propensities as

$$
\begin{aligned}
& a_{1}^{f}(\mathbf{x})=k_{1}^{f} n_{A}, \\
& a_{1}^{r}(\mathbf{x})=k_{1}^{r} n_{B}, \\
& a_{2}^{f}(\mathbf{x})=k_{2}^{f} n_{C} .
\end{aligned}
$$


If $k_{1}^{f}, k_{1}^{r} \gg k_{2}^{f}$, then we can partition $\epsilon_{1}$ as the fast-reaction extent $\mathbf{z}$ and $\epsilon_{2}$ as the slow-reaction extent $\mathbf{y}$. Additionally, we would expect the fast extent of reaction to equilibrate (relax) before the expected time to the first slow reaction. Returning to the master equation formalism, this equilibration implies that we should approximate the fast reactions, Eq. (27b), as

$$
\begin{aligned}
0 \approx & \sum_{k=1}^{l} c_{k}^{f}\left(\mathbf{y}, \mathbf{z}-\boldsymbol{I}_{k}^{z}\right) P_{A}\left(\mathbf{z}-\boldsymbol{I}_{k}^{z} \mid \mathbf{y} ; t\right) \\
& +c_{k}^{r}\left(\mathbf{y}, \mathbf{z}+\boldsymbol{I}_{k}^{z}\right) P_{A}\left(\mathbf{z}+\boldsymbol{I}_{k}^{z} \mid \mathbf{y} ; t\right) \\
& -\left(c_{k}^{f}(\mathbf{y}, \mathbf{z})+c_{k}^{r}(\mathbf{y}, \mathbf{z})\right) P_{A}(\mathbf{z} \mid \mathbf{y} ; t) \\
& \forall \mathbf{y} \in \mathbb{Y}, \quad \mathbf{z} \in \mathbb{Z} .
\end{aligned}
$$

Here, we expect that $\mathbb{Z}_{p}=\mathbb{Z}$ and $Y_{p}=Y$ since fast reactions always have large propensities for this case. The resulting coupled master equations are

$$
\begin{aligned}
& \frac{d P(\mathbf{y} ; t)}{d t} \approx \sum_{j=1}^{m-l}\left(\sum_{\mathbf{z} \in Z_{p}} b_{j}^{f}\left(\mathbf{y}-\boldsymbol{I}_{j}^{y}, \mathbf{z}\right) P_{A}\left(\mathbf{z} \mid \mathbf{y}-\boldsymbol{I}_{j}^{y} ; t\right)\right) P\left(\mathbf{y}-\boldsymbol{I}_{j}^{y} ; t\right) \\
&+\left(\sum_{\mathbf{z} \in Z_{p}} b_{j}^{r}\left(\mathbf{y}+\boldsymbol{I}_{j}^{y}, \mathbf{z}\right) P_{A}\left(\mathbf{z} \mid \mathbf{y}+\boldsymbol{I}_{j}^{y} ; t\right)\right) P\left(\mathbf{y}+\boldsymbol{I}_{j}^{y} ; t\right) \\
&-\left(\sum_{\mathbf{z} \in Z_{p}}\left(b_{j}^{f}(\mathbf{y}, \mathbf{z})+b_{j}^{r}(\mathbf{y}, \mathbf{z})\right) P_{A}(\mathbf{z} \mid \mathbf{y} ; t)\right) P(\mathbf{y} ; t) \\
& \forall \mathbf{y} \in \mathbb{Y}, \\
& \\
& \sum_{k=1} c_{k}^{f}\left(\mathbf{y}, \mathbf{z}-\boldsymbol{I}_{k}^{z}\right) P_{A}\left(\mathbf{z}-\boldsymbol{I}_{k}^{z} \mid \mathbf{y} ; t\right) \\
&+ c_{k}^{r}\left(\mathbf{y}, \mathbf{z}+\boldsymbol{I}_{k}^{z}\right) P_{A}\left(\mathbf{z}+\boldsymbol{I}_{k}^{z} \mid \mathbf{y} ; t\right) \\
&-\left(c_{k}^{f}(\mathbf{y}, \mathbf{z})+c_{k}^{r}(\mathbf{y}, \mathbf{z})\right) P_{A}(\mathbf{z} \mid \mathbf{y} ; t) \\
& \forall \mathbf{y} \in \mathbb{Y}, \quad \mathbf{z} \in \mathbb{Z} .
\end{aligned}
$$

This coupled system, Eq. (31), is comparable to that proposed by Haken, ${ }^{13}$ who considered the adiabatic elimination of fast-relaxing variables from the discrete master equation. In contrast to Haken's derivation, we have chosen a specific coordinate system (the net extent of reaction) and a specific means of partitioning this coordinate (by the order of magnitude of the corresponding reaction propensities). Given these specific choices, inspection of Eq. (14) reveals that the special nature of the stoichiometric matrix for the extent system (an identity matrix) zeros the sum

$$
\begin{aligned}
0= & \sum_{\mathbf{z} \in \mathbb{Z}_{p}} \sum_{k=1}^{l} c_{k}^{f}\left(\mathbf{y}, \mathbf{z}-\boldsymbol{I}_{k}^{z}\right) P\left(\mathbf{y}, \mathbf{z}-\boldsymbol{I}_{k}^{z} ; t\right) \\
& +c_{k}^{r}\left(\mathbf{y}, \mathbf{z}+\boldsymbol{I}_{k}^{z}\right) P\left(\mathbf{y}, \mathbf{z}+\boldsymbol{I}_{k}^{z} ; t\right) \\
& -\left(c_{k}^{f}(\mathbf{y}, \mathbf{z})+c_{k}^{r}(\mathbf{y}, \mathbf{z})\right) P(\mathbf{y}, \mathbf{z} ; t) \\
& \forall \mathbf{y} \in \mathbb{Y}_{p} .
\end{aligned}
$$

That is, this coordinate system generates the same value of $\mathbf{y}$ in all terms of Eq. (32) when summing over $\mathbf{z} \in \mathbb{Z}_{p}$, thus making the sum over all fast-reaction propensities zero. Hence our specific choices lead to significant simplification for the evolution equation of the slow marginal density, a result that is not obvious from Haken's derivation. Additionally, Haken's adiabatic hypothesis makes the assumption that fast transitions do not affect the slow partitioned variables. Here, we demonstrate that choosing the appropriate coordinate and partitioning strategy enforces this particular constraint; in this case, fast reactions do not change the value of the slow-reaction extents.

This coupled system, Eq. (31), is markedly similar to the governing equations for the slow-scale simulation recently proposed by Cao et al. ${ }^{16}$ Their derivation is different from ours, however, and the differences deserve some attention. First, Cao et al. ${ }^{16}$ partition on the basis of fast and slow species rather than extents, with fast species affected by at least one fast reaction and slow species affected by solely slow reactions. We have chosen to remain in the extent space because reactions are equilibrating, not chemical species. Indeed, Cao et al. ${ }^{16}$ note that reactions (not chemical species) are the primary concern when they pose the question: "[I]s there a legitimate way to skip over the fast reactions and explicitly simulate only the slow reactions?" Also, Cao et al. ${ }^{16}$ use the construct of a virtual fast system to arrive at an evolution equation for the slow species [similar to our evolution equation for the slow-extent marginal, Eq. (15)], a choice that obviates the need for defining an evolution equation for the conditional density $P(\mathbf{z} \mid \mathbf{y})$. In contrast to this approach, our approach has a tighter connection to the original master equation because we derived the coupled system, Eq. (31), directly from the original master equation and because we can obtain an approximate value of the joint density $P(\mathbf{y}, \mathbf{z} ; t)$ through Eq. (26). Also, all approximations arise directly from order-of-magnitude and relaxation-time arguments.

We note that the evolution equation for the slow species derived by Cao et al. ${ }^{16}$ can also be derived using conditional density arguments. We consider partitioning the species in the same manner as these authors, denoting the slow species as $\mathbf{n}^{s}$ and the fast species as $\mathbf{n}^{f}$. We define the partitioned subsets of the fast and slow species as $\mathbb{N}_{p}^{f}$ and $\mathbb{N}_{p}^{s}$, respectively. The stoichiometric matrix for the species coordinate partitions as follows:

$$
\boldsymbol{\nu}=\left[\begin{array}{ll}
\overline{\boldsymbol{\nu}}^{s} & \overline{\boldsymbol{\nu}}^{f} \\
0 & \underline{\boldsymbol{\nu}}^{f}
\end{array}\right] .
$$

Then the corresponding evolution equation for the slow marginal density is 


$$
\begin{aligned}
& \frac{d P\left(\mathbf{n}^{s} ; t\right)}{d t}=\sum_{j=1}^{m-l} \sum_{\mathbf{n}^{f} \in \mathbb{N}_{p}^{f}} b_{j}^{f}\left(\mathbf{n}^{s}-\overline{\boldsymbol{\nu}}_{j}^{s}, \mathbf{n}^{f}-\overline{\boldsymbol{\nu}}_{j}^{f}\right) P\left(\mathbf{n}^{s}-\overline{\boldsymbol{\nu}}_{j}^{s}, \mathbf{n}^{f}-\overline{\boldsymbol{\nu}}_{j}^{f} ; t\right)+b_{j}^{r}\left(\mathbf{n}^{s}+\overline{\boldsymbol{\nu}}_{j}^{s}, \mathbf{n}^{f}+\overline{\boldsymbol{\nu}}_{j}^{f}\right) P\left(\mathbf{n}^{s}+\overline{\boldsymbol{\nu}}_{j}^{s}, \mathbf{n}^{f}+\overline{\boldsymbol{\nu}}_{j}^{f} ; t\right) \\
& -\left(b_{j}^{f}\left(\mathbf{n}^{s}, \mathbf{n}^{f}\right)+b_{j}^{r}\left(\mathbf{n}^{s}, \mathbf{n}^{f}\right)\right) P\left(\mathbf{n}^{s}, \mathbf{n}^{f} ; t\right) \\
& +\sum_{k=1}^{l} \sum_{\mathbf{n}^{f} \in \mathbb{N}_{p}^{f}} c_{k}^{f}\left(\mathbf{n}^{s}, \mathbf{n}^{f}-\underline{\boldsymbol{v}}_{k}^{f}\right) P\left(\mathbf{n}^{s}, \mathbf{n}^{f}-\underline{\boldsymbol{v}}_{k}^{f} ; t\right)+c_{k}^{r}\left(\mathbf{n}^{s}, \mathbf{n}^{f}+\underline{\boldsymbol{v}}_{k}^{f}\right) P\left(\mathbf{n}^{s}, \mathbf{n}^{f}+\underline{\boldsymbol{v}}_{k}^{f} ; t\right)-\left(c_{k}^{f}\left(\mathbf{n}^{s}, \mathbf{n}^{f}\right)+c_{k}^{r}\left(\mathbf{n}^{s}, \mathbf{n}^{f}\right)\right) P\left(\mathbf{n}^{s}, \mathbf{n}^{f} ; t\right) \\
& =\sum_{j=1}^{m-l}\left[\left(\sum_{\mathbf{n}^{f} \in \mathbb{N}_{p}^{f}} b_{j}^{f}\left(\mathbf{n}^{s}-\overline{\boldsymbol{\nu}}_{j}^{s}, \mathbf{n}^{f}-\overline{\boldsymbol{\nu}}_{j}^{f}\right) P\left(\mathbf{n}^{f}-\overline{\boldsymbol{\nu}}_{j}^{f} \mid \mathbf{n}^{s}-\overline{\boldsymbol{\nu}}_{j}^{s} ; t\right)\right) P\left(\mathbf{n}^{s}-\overline{\boldsymbol{\nu}}_{j}^{s} ; t\right)\right. \\
& \left.+\left(\sum_{\mathbf{n}^{f} \in \mathbb{N}_{p}^{f}} b_{j}^{r}\left(\mathbf{n}^{s}+\overline{\boldsymbol{\nu}}_{j}^{s}, \mathbf{n}^{f}+\overline{\boldsymbol{\nu}}_{j}^{f}\right) P\left(\mathbf{n}^{f}+\overline{\boldsymbol{\nu}}_{j}^{f} \mid \mathbf{n}^{s}+\overline{\boldsymbol{\nu}}_{j}^{s} ; t\right)\right) P\left(\mathbf{n}^{s}+\overline{\boldsymbol{\nu}}_{j}^{s} ; t\right)-\left(\sum_{\mathbf{n}^{f} \in \mathbb{N}_{p}^{f}}\left(b_{j}^{f}\left(\mathbf{n}^{s}, \mathbf{n}^{f}\right)+b_{j}^{r}\left(\mathbf{n}^{s}, \mathbf{n}^{f}\right)\right) P\left(\mathbf{n}^{f} \mid \mathbf{n}^{s} ; t\right)\right) P\left(\mathbf{n}^{s} ; t\right)\right]
\end{aligned}
$$

in which the fast reactions have again dropped out of the evolution equation. Additionally, we can use order-ofmagnitude arguments to derive an approximate evolution equation for the conditional density $P\left(\mathbf{n}^{f} \mid \mathbf{n}^{s} ; t\right)$ that is analogous to Eq. (31b). Hence the governing equations for the slow-scale simulation can also arise from conditional density arguments.

\section{E. The Langevin and deterministic approximations}

We now consider the case in which both fast and slow extents relax at similar time scales. Revisiting the reaction example (28), we consider the case in which $k_{1}^{f} \gg k_{1}^{r}, k_{2}^{f}$ and $n_{A o} \gg n_{B o}, n_{C o}$ in which the notation $n_{A o}$ refers to the initial number of $A$ molecules. For this example, we partition $\epsilon_{1}$ as the fast-reaction extent $\mathbf{z}$ and $\epsilon_{2}$ as the slow-reaction extent y. Until a significant amount of $A$ has been consumed, we would expect numerous firings of reaction (1) interspersed with relatively few firings of reaction (2). Clearly the system never equilibrates, but rather fast and slow reactions fire until the fast-reaction propensity reaches a similar order of magnitude as the slow-reaction propensity. We note that the net extent coordinate system requires us to either neglect the reverse reaction of $\epsilon_{1}$ or to treat it the same as the forward reaction of $\epsilon_{1}$ even though its propensity may be the same order of magnitude as that of the slow extent [i.e., $a_{1}^{f}(\mathbf{x})$ $\gg a_{1}^{r}(\mathbf{x}), a_{2}^{f}(\mathbf{x})$ and $\left.a_{1}^{r}(\mathbf{x}) \approx a_{2}^{f}(\mathbf{x})\right]$. However, we expect such approximations to introduce a negligible amount of error into the estimates of the net extents. Note also that, in contrast to the equilibrium approximation, we have introduced the number of molecules into the time-scale argument. For most cases, we expect this time-scale argument to involve large numbers of reacting molecules, but such involvement is not always the case as demonstrated in the viral infection example presented by Haseltine and Rawlings. ${ }^{17}$ Rather, we require that the magnitude of the fast-reaction propensities remain large relative to the magnitude of the slowreaction propensities through the expected time of the first slow reaction.
Returning to the master equation formalism, this process requires a different approximation for the conditional density $P(\mathbf{z} \mid \mathbf{y})$. We proceed by demonstrating as outlined by Gardiner $^{25}$ how this subset can be approximated using the Langevin approximation. Define the characteristic size of the system to be $\Omega$ and use this size to recast the master equation (25) in terms of intensive variables (let $\boldsymbol{Z} \leftarrow \mathbf{z} / \Omega$ ). Performing a Kramers-Moyal expansion on this master equation results in a system size expansion in $\Omega$. In the limit as $\mathbf{z}$ and $\Omega$ become large, the discrete master equation (27b) can be approximated by its first two differential moments with the continuous Fokker-Planck equation

$$
\begin{aligned}
\frac{\partial P_{A}(\mathbf{z} \mid \mathbf{y} ; t)}{\partial t}= & -\sum_{i=1}^{l} \frac{\partial}{\partial \mathbf{z}_{i}}\left(\boldsymbol{A}_{i}(\mathbf{y}, \mathbf{z}) P_{A}(\mathbf{z} \mid \mathbf{y} ; t)\right) \\
& +\frac{1}{2} \sum_{i=1}^{l} \sum_{j=1}^{l} \frac{\partial^{2}}{\partial \mathbf{z}_{i} \partial \mathbf{z}_{j}}\left(\boldsymbol{B}_{i j}(\mathbf{y}, \mathbf{z})^{2} P_{A}(\mathbf{z} \mid \mathbf{y} ; t)\right) \\
& \forall \mathbf{y} \in \mathbb{Y}_{p}, \quad \mathbf{z} \in \mathbb{Z}_{p}
\end{aligned}
$$

in which (noting that $\mathbf{z}$ consists of extents of reaction)

$$
\begin{aligned}
\boldsymbol{A}(\mathbf{y}, \mathbf{z})= & \sum_{i=1}^{l} \boldsymbol{I}_{i}^{z}\left(c_{i}^{f}(\mathbf{y}, \mathbf{z})-c_{i}^{r}(\mathbf{y}, \mathbf{z})\right) \\
= & {\left[c_{1}^{f}(\mathbf{y}, \mathbf{z})-c_{1}^{r}(\mathbf{y}, \mathbf{z}), c_{2}^{f}(\mathbf{y}, \mathbf{z})-c_{2}^{r}(\mathbf{y}, \mathbf{z}), \ldots,\right.} \\
& \left.c_{l}^{f}(\mathbf{y}, \mathbf{z})-c_{l}^{r}(\mathbf{y}, \mathbf{z})\right]^{T}, \\
{[\boldsymbol{B}(\mathbf{y}, \mathbf{z})]^{2}=} & \sum_{i=1}^{l} \boldsymbol{I}_{i}^{z}\left(\boldsymbol{I}_{i}^{z}\right)^{T}\left(c_{i}^{f}(\mathbf{y}, \mathbf{z})-c_{i}^{r}(\mathbf{y}, \mathbf{z})\right) \\
= & \operatorname{diag}\left(c_{1}^{f}(\mathbf{y}, \mathbf{z})+c_{1}^{r}(\mathbf{y}, \mathbf{z}),\right. \\
& \left.c_{2}^{f}(\mathbf{y}, \mathbf{z})+c_{2}^{r}(\mathbf{y}, \mathbf{z}), \ldots, c_{l}^{f}(\mathbf{y}, \mathbf{z})+c_{l}^{r}(\mathbf{y}, \mathbf{z})\right) .
\end{aligned}
$$

Here, $\operatorname{diag}(a, \ldots, z)$ defines a matrix with elements $a, \ldots, z$ on the diagonal. Equation (36) has Itô solution of the form 


$$
\begin{aligned}
d \mathbf{z}_{i} & =\boldsymbol{A}_{i}(\mathbf{y}, \mathbf{z}) d t+\sum_{j=1}^{l} \boldsymbol{B}_{i j}(\mathbf{y}, \mathbf{z}) d \boldsymbol{W}_{j}, \\
& \forall 1 \leqslant i \leqslant l, \mathbf{y} \in \mathbb{Y}_{p}, \quad \mathbf{z} \in \mathbb{Z}_{p} \\
& =\left(c_{i}^{f}(\mathbf{y}, \mathbf{z})-c_{i}^{r}(\mathbf{y}, \mathbf{z})\right) d t+\sqrt{c_{i}^{f}(\mathbf{y}, \mathbf{z})+c_{i}^{r}(\mathbf{y}, \mathbf{z})} d \boldsymbol{W}_{i} \\
& \forall 1 \leqslant i \leqslant l, \quad \mathbf{y} \in \mathbb{Y}_{p}, \quad \mathbf{z} \in \mathbb{Z}_{p}
\end{aligned}
$$

in which $\boldsymbol{W}$ is a vector of Wiener processes. Equation (41) is the chemical Langevin equation, whose formulation was recently readdressed by Gillespie. ${ }^{26}$ Note the difference between Eqs. (36) and (41). The Fokker-Planck equation (36) specifies the distribution of the stochastic process, whereas the stochastic differential equation (41) specifies how the trajectories of the state evolve. Also, bear in mind that whether or not a given $\Omega$ is large enough to permit truncation of the system size, expansion is relative. In this case, $\Omega$ is of sufficient magnitude to make this approximation valid for only a subset of the reactions, not the entire system.

Combining the evolution equations for the slow- and fast-reaction extents, i.e., Eqs. (27a) and (36), respectively, the problem of interest is the coupled set of master equations

$$
\begin{aligned}
& \frac{d P(\mathbf{y} ; t)}{d t} \\
& \approx \sum_{k=1}^{m-l}\left(\int_{\mathbf{z}} b_{k}^{f}\left(\mathbf{y}-\boldsymbol{I}_{k}^{y}, \mathbf{z}_{k}^{\prime}\right) P_{A}\left(\mathbf{z}_{k}^{\prime} \mid \mathbf{y}-\boldsymbol{I}_{k}^{y} ; t\right) d \mathbf{z}^{\prime}\right) P\left(\mathbf{y}-\boldsymbol{I}_{k}^{y} ; t\right) \\
& +\sum_{k=1}^{m-l}\left(\int_{\mathbf{z}} b_{k}^{r}\left(\mathbf{y}+\boldsymbol{I}_{k}^{y}, \mathbf{z}_{k}^{\prime}\right) P_{A}\left(\mathbf{z}_{k}^{\prime} \mid \mathbf{y}+\boldsymbol{I}_{k}^{y} ; t\right) d \mathbf{z}^{\prime}\right) P\left(\mathbf{y}+\boldsymbol{I}_{k}^{y} ; t\right) \\
& -\left(\int_{\mathbf{z}}\left(b_{k}^{f}\left(\mathbf{y}, \mathbf{z}^{\prime}\right)+b_{k}^{r}\left(\mathbf{y}, \mathbf{z}^{\prime}\right)\right) P_{A}\left(\mathbf{z}^{\prime} \mid \mathbf{y} ; t\right) d \mathbf{z}^{\prime}\right) P(\mathbf{y} ; t) \\
& \forall \mathbf{y} \in \mathbb{Y}_{p}, \\
& \frac{\partial P_{A}(\mathbf{z} \mid \mathbf{y} ; t)}{\partial t}=-\sum_{i=1}^{l} \frac{\partial}{\partial \mathbf{z}_{i}}\left(\boldsymbol{A}_{i}(\mathbf{y}, \mathbf{z}) P_{A}(\mathbf{z} \mid \mathbf{y} ; t)\right) \\
& +\frac{1}{2} \sum_{i=1}^{l} \sum_{j=1}^{l} \frac{\partial^{2}}{\partial \mathbf{z}_{i} \partial \mathbf{z}_{j}}\left(\boldsymbol{B}_{i j}(\mathbf{y}, \mathbf{z})^{2} P_{A}(\mathbf{z} \mid \mathbf{y} ; t)\right) \\
& \forall \mathbf{y} \in \mathbb{Y}_{p}, \quad \mathbf{z}_{i} \in \mathbb{Z}_{p}, \\
& \frac{m P(\mathbf{x} ; t)}{d t}=\sum_{k=1}^{m} a_{k}^{f}\left(\mathbf{x}-\boldsymbol{I}_{k}\right) P\left(\mathbf{x}-\boldsymbol{I}_{k} ; t\right) \\
& +a_{k}^{r}\left(\mathbf{x}+\boldsymbol{I}_{k}\right) P\left(\mathbf{x}+\boldsymbol{I}_{k} ; t\right)-\left(a_{k}^{f}(\mathbf{x})+a_{k}^{r}(\mathbf{x})\right) P(\mathbf{x} ; t) \\
& \forall \mathbf{x} \notin \mathbb{X}_{p} .
\end{aligned}
$$

If we can solve these equations simultaneously, then we have an approximate solution to the original master equation (8) due to the definition of the conditional density given by Eq. (26). Note that the solution is approximate because we have used the Fokker-Planck approximation for the master equation of the fast reactions.

In the thermodynamic limit $(\mathbf{z} \rightarrow \infty, \Omega \rightarrow \infty, Z=\mathbf{z} / \Omega$ $=$ finite), the intensive variables for the fast subset of reactions (Z's) evolve deterministically. ${ }^{27}$ Accordingly, we propose further approximating the Langevin equation (41) as

$d \mathbf{z}_{i}=\left(c_{i}^{f}(\mathbf{y}, \mathbf{z})-c_{i}^{r}(\mathbf{y}, \mathbf{z})\right) d t \quad \forall 1 \leqslant i \leqslant l, \quad \mathbf{y} \in \mathbb{Y}_{p}, \quad \mathbf{z} \in \mathbb{Z}_{p}$.

In this case, the coupled master equations (42) reduce to

$$
\begin{aligned}
\frac{d P(\mathbf{y} ; t)}{d t} \approx & \sum_{k=1}^{m-l} b_{k}^{f}\left(\mathbf{y}-\boldsymbol{I}_{k}^{y}, \mathbf{z}(t)\right) P\left(\mathbf{y}-\boldsymbol{I}_{k}^{y} ; t\right) \\
& +b_{k}^{r}\left(\mathbf{y}+\boldsymbol{I}_{k}^{y}, \mathbf{z}(t)\right) P\left(\mathbf{y}+\boldsymbol{I}_{k}^{y} ; t\right) \\
& -\left(b_{k}^{f}(\mathbf{y}, \mathbf{z}(t))+b_{k}^{r}(\mathbf{y}, \mathbf{z}(t))\right) P(\mathbf{y} ; t) \\
& \forall \mathbf{y} \in \mathbb{Y}_{p},
\end{aligned}
$$

$$
d \mathbf{z}_{i}=\left(c_{i}^{f}(\mathbf{y}, \mathbf{z})-c_{i}^{r}(\mathbf{y}, \mathbf{z})\right) d t \quad \forall 1 \leqslant i \leqslant l, \quad \mathbf{y} \in \mathbb{Y}_{p}, \quad \mathbf{z} \in \mathbb{Z}_{p},
$$

$$
\begin{aligned}
\frac{d P(\mathbf{x} ; t)}{d t}= & \sum_{k=1}^{m} a_{k}^{f}\left(\mathbf{x}-\boldsymbol{I}_{k}\right) P\left(\mathbf{x}-\boldsymbol{I}_{k} ; t\right)+a_{k}^{r}\left(\mathbf{x}+\boldsymbol{I}_{k}\right) P\left(\mathbf{x}+\boldsymbol{I}_{k} ; t\right) \\
& -\left(a_{k}^{f}(\mathbf{x})+a_{k}^{r}(\mathbf{x})\right) P(\mathbf{x} ; t) \quad \forall \mathbf{x} \notin \mathbb{X}_{p},
\end{aligned}
$$

in which $\mathbf{z}(t)$ is the solution to the differential equation (43). The benefit of this assumption is that Eq. (43) can be solved rigorously using an ordinary differential equation (ODE) solver. If the magnitude of the fluctuations in this term is small compared to the sensitivity of $c_{i}(\mathbf{y}, \mathbf{z})$ to the subset $\mathbf{y}$, then Eq. (43) is a valid approximation. This approximation is also valid if one is primarily concerned with the fluctuations in the small-numbered species as opposed to the largenumbered species, assuming that the extents approximated by Eq. (43) predominantly affect the population size of largenumbered species. Alternatively, we could recast the extensive variables (i.e., $\mathbf{x}, \mathbf{y}$, and $\mathbf{z}$ ) as intensive variables; in this case, we would expect smaller deviations between the original and partitioned master equations.

This derivation points out several weaknesses in our previous justification of the hybrid system. ${ }^{17}$ Previously, we derived an approximate expression for the fast marginal density $P(\mathbf{z} ; t)$ using the order-of-magnitude argument

$$
\frac{d P(\mathbf{y} \mid \mathbf{z} ; t)}{d t} \approx 0
$$


We also postulated (without derivation) the functional form of the evolution equation for the slow marginal density $P(\mathbf{y} ; t)$. The postulated evolution equation is virtually identical to Eq. (44), but with a different coordinate (irreversible as opposed to net extent). Here, we derived the evolution equation for the slow marginal density $P(\mathbf{y} ; t)$ and the (approximate) conditional density $P_{A}(\mathbf{z} \mid \mathbf{y} ; t)$ using more precise order-of-magnitude arguments [i.e., Eq. (5)]. By so doing, we have the stronger connection between the approximated densities and the original joint density through Eq. (26).

\section{NUMERICAL IMPLEMENTATION OF THE APPROXIMATIONS}

We now outline procedures for implementing the equilibrium, Langevin, and deterministic approximations presented in Sec. II. We propose using simulation to reconstruct moments of the underlying master equation. We note that the simulation requires that all reversible reactions be treated as two irreversible reactions. Consequently, we group all of the reversible reaction propensities together for the unpartitioned reactions and the slow-partitioned reactions, i.e.,

$$
\left[\begin{array}{c}
a_{1}^{f}(x) \\
a_{1}^{r}(x) \\
\vdots \\
a_{m}^{f}(x) \\
a_{m}^{r}(x)
\end{array}\right]=\left[\begin{array}{c}
a_{1}(x) \\
a_{2}(x) \\
\vdots \\
a_{2 m-1}(x) \\
a_{2 m}(x)
\end{array}\right]
$$

and

$$
\left[\begin{array}{c}
b_{1}^{f}(\mathbf{y}, \mathbf{z}) \\
b_{1}^{r}(\mathbf{y}, \mathbf{z}) \\
\vdots \\
b_{m-l}^{f}(\mathbf{y}, \mathbf{z}) \\
b_{m-l}^{r}(\mathbf{y}, \mathbf{z})
\end{array}\right]=\left[\begin{array}{c}
b_{1}(\mathbf{y}, \mathbf{z}) \\
b_{2}(\mathbf{y}, \mathbf{z}) \\
\vdots \\
b_{2 m-2 l-1}(\mathbf{y}, \mathbf{z}) \\
b_{2 m-2 l}(\mathbf{y}, \mathbf{z})
\end{array}\right]
$$

For the slow reactions, Gillespie ${ }^{28}$ outlines a general method for exact stochastic simulation that is applicable to the desired problem, Eq. (27a). This method examines the joint probability function, $P(\tau, \mu)$, that governs when the next reaction occurs and which reaction occurs. For this end, we define

$$
\overline{b_{\mu}(\mathbf{y}, \mathbf{z} ; t)} d t= \begin{cases}\sum_{\mathbf{z} \in Z_{p}} b_{\mu}(\mathbf{y}, \mathbf{z}) P_{A}(\mathbf{z} \mid \mathbf{y} ; t) d t \quad \text { equilibrium approximation } \\ \int_{\mathbf{z}} b_{\mu}\left(\mathbf{y}, \mathbf{z}^{\prime}\right) P_{A}\left(\mathbf{z}^{\prime} \mid \mathbf{y} ; t\right) d \mathbf{z}^{\prime} d t \quad \text { Langevin or deterministic approximation }\end{cases}
$$

in which $\overline{b_{\mu}(\mathbf{y}, \mathbf{z} ; t)} d t$ is the probability (first order in $d t$ ) that reaction $\mu$ occurs in the next time interval $d t$. The joint probability function $P(\tau, \mu)$ is

$$
P(\tau, \mu)=\overline{b_{\mu}(\mathbf{y}, \mathbf{z} ; t+\tau)} \exp \left(-\int_{t}^{t+\tau} r_{\text {tot }}^{y}\left(t^{\prime}\right) d t^{\prime}\right)
$$

in which

$$
r_{\text {tot }}^{y}(t)=\sum_{j=1}^{2 m-2 l} \overline{b_{j}(\mathbf{y}, \mathbf{z} ; t)} .
$$

We refer the interested reader to Haseltine ${ }^{18}$ for details on the derivation of this function.

By conditioning the joint probability function $P(\tau, \mu)$,

$$
P(\tau, \mu)=P(\mu \mid \tau) P(\tau),
$$

we can determine the probability density for when the next reaction occurs

$$
P(\tau)=r_{\mathrm{tot}}^{y}(t+\tau) \exp \left(-\int_{t}^{t+\tau} r_{\mathrm{tot}}^{y}\left(t^{\prime}\right) d t^{\prime}\right)
$$

and which reaction it will be

$$
P(\mu \mid \tau)=\frac{\overline{b_{\mu}(\mathbf{y}, \mathbf{z} ; t+\tau)}}{r_{\mathrm{tot}}^{y}(t+\tau)} .
$$

These manipulations correspond to the hybrid equivalent of the direct method. It is a simple exercise left to derive the necessary densities [i.e., $P(\tau \mid \mu)$ and $P(\mu)$ ] for the hybrid equivalents of the first and next reaction method. Using Monte Carlo simulation, we obtain realizations of the desired joint probability function $P(\tau, \mu)$ by randomly selecting $\tau$ and $\mu$ from the probability densities defined by Eqs. (50) and (51). Given two random numbers $p_{1}$ and $p_{2}$ uniformly distributed on $(0,1), \tau$ and $\mu$ are constrained accordingly:

$$
\int_{t}^{t+\tau} r_{\mathrm{tot}}^{y}\left(t^{\prime}\right) d t^{\prime}+\log \left(p_{1}\right)=0,
$$




$$
\sum_{k=1}^{\mu-l} \overline{b_{k}(\mathbf{y}, \mathbf{z} ; t+\tau)}<p_{2} r_{\text {tot }}^{y}(t+\tau) \leqslant \sum_{k=1}^{\mu} \overline{b_{k}(\mathbf{y}, \mathbf{z} ; t+\tau)}
$$

Simulating the different approximations require slightly different algorithms, which we address next.

\section{A. Simulating the equilibrium approximation}

We first address the equilibrium approximation. For this case,

$$
\overline{b_{j}(\mathbf{y}, \mathbf{z} ; t)}=\sum_{\mathbf{z} \in \mathbb{Z}} b_{j}(\mathbf{y}, \mathbf{z}) P_{A}(\mathbf{z} \mid \mathbf{y} ; t) \quad \forall 1 \leqslant j \leqslant 2 m-2 l .
$$

Additionally, the quantities $\overline{b_{j}(\mathbf{y}, \mathbf{z} ; t)}$ are actually time invariant between slow reactions. Thus, the integral constraint (52a) reduces to the algebraic relation

$$
\tau=-\frac{\log \left(p_{1}\right)}{r_{\text {tot }}^{y}(t)} \text {. }
$$

Algorithm 1 presents one method of solving this system. Note that we could draw a sample from the equilibrium distribution $P_{A}(\mathbf{z} \mid \mathbf{y})$ at any time to determine a current value of the state, which may be desirable for sampling the system at uniform time increments. Also, this algorithm is similar to the slow-scale stochastic simulation algorithm proposed by Cao et al., ${ }^{16}$ with the exception that our algorithm partitions extents as opposed to species.
Solution of the equilibrated density $P_{A}(\mathbf{z} \mid \mathbf{y})$ deserves some further attention. If we stack probabilities for all possible values of the fast extents into a vector $\mathbf{p}$, we can recast the continuous-time master equation as a vector-matrix problem, i.e.,

$$
\frac{d \mathbf{p}}{d t}=\boldsymbol{C} \mathbf{p} \approx 0 \quad \text { (equilibrium assumption) }
$$

in which $\boldsymbol{C}$ is the matrix of reaction propensities. The equilibrium distribution is then the null space of the matrix $\boldsymbol{C}$, which we can compute numerically. In general, we expect $\boldsymbol{C}$ to be a sparse matrix. Consequently, we can efficiently solve the linear system (55) for $\mathbf{p}$ using Krylov iterative methods ${ }^{29}$ such as the biconjugate gradient-stabilized method. Cao et al. ${ }^{16}$ outline some alternative, approximate methods for evaluating this equilibrated density.

\section{B. Simulating the Langevin and deterministic approximations: Exact next-reaction time}

We now address methods for simulating the Langevin and deterministic approximations. These approximations have time-varying reaction propensities, so we must satisfy Eq. (52a) by integrating $r_{\text {tot }}^{y}$ and the fast subset of reactions $\mathbf{z}$ forward in time until the following conditions are met:

Off-line. Partition the set $\mathbf{x}$ of $2 m$ extents of reaction into fast and slow extents. Determine the partitioned stoichiometric matrices [the matrices $\boldsymbol{\nu}^{y}$ and $\boldsymbol{\nu}^{z}$ ] and the reaction propensity laws $\left[a_{k}(\mathbf{y}, \mathbf{z})^{\text {'s }}\right]$. Also, choose a strategy for solving the distribution $P_{A}(\mathbf{z} \mid \mathbf{y})$ given by Eq. (31b) for the fast reactions in the partitioned case.

Initialize. Set the time $t$ equal to zero.

Set the number of species $\mathbf{n}$ to $\mathbf{n}_{0}$.

1. Solve for the distribution $P_{A}(\mathbf{z} \mid \mathbf{y})$, denoting all possible combinations of $\mathbf{z}$ as $(\mathbf{z}(0), \ldots, \mathbf{z}(t))$.

Record the initial value of $\mathbf{z}$ as $\mathbf{z}(i)$.

2. For subset $\mathbf{y}$, calculate

(a) the reaction propensities, $\overline{b_{j}(\mathbf{y}, \mathbf{z})}=\sum_{\mathbf{z} \in \mathbb{Z}} b_{j}(\mathbf{y}, \mathbf{z}) P_{A}(\mathbf{z} \mid \mathbf{y}) \forall j=1, \ldots, 2 m-2 l$, and

(b) the total reaction propensity, $r_{\text {tot }}^{y}=\sum_{k=1}^{2 m-2 l} \overline{b_{k}(\mathbf{y}, \mathbf{z})}$.

3. Select three random numbers $p_{1}, p_{2}$, and $p_{3}$ from the uniform distribution $(0,1)$.

4. Choose $\mathbf{z}(j)$ from the distribution $P_{A}(\mathbf{z} \mid \mathbf{y})$ such that

$$
\sum_{k=1}^{j-1} P_{A}(\mathbf{z}(k) \mid \mathbf{y})<p_{1} \leqslant \sum_{k=1}^{j} P_{A}(\mathbf{z}(k) \mid \mathbf{y}) .
$$

Set $\hat{\boldsymbol{\nu}}_{\mathbf{z}}=\mathbf{z}(j)-\mathbf{z}(i)$.

5. Let $\tau=-\log \left(p_{2}\right) / r_{\text {tot }}^{y}$. Choose $j$ such that

$$
\sum_{k=1}^{j-1} \overline{b_{k}(\mathbf{y}, \mathbf{z})}<p_{3} r_{\text {tot }}^{y} \leqslant \sum_{k=1}^{j} \overline{b_{k}(\mathbf{y}, \mathbf{z})}
$$

6. Let $\mathbf{n} \leftarrow \mathbf{n}+\left(\boldsymbol{\nu}_{j}^{y}\right)^{T}+\hat{\boldsymbol{\nu}}_{\mathbf{z}}$, where $\boldsymbol{\nu}_{j}^{y}$ is the $j$ th row of $\boldsymbol{\nu}^{y}$.

Go to step 1. 
$\int_{t}^{t+\tau} r_{\mathrm{tot}}^{y}\left(t^{\prime}\right) d t^{\prime}+\log \left(p_{1}\right)=0$

$r_{\mathrm{tot}}^{y}(t)=\sum_{j=1}^{2 m-2 l} \overline{b_{j}(\mathbf{y}, \mathbf{z} ; t)}$

$\overline{b_{j}(\mathbf{y}, \mathbf{z} ; t)}=\int_{z} b_{j}\left(\mathbf{y}, \mathbf{z}^{\prime}\right) P_{A}\left(\mathbf{z}^{\prime} \mid \mathbf{y} ; t\right) d \mathbf{z}^{\prime} \quad \forall 1 \leqslant j \leqslant 2 m-2 l$.

For the Langevin approximation, we propose reconstructing the density $P_{A}(\mathbf{z} \mid \mathbf{y} ; t)$ by simulating the stochastic differential equation (41) (also known as the Langevin equation). In this case, Eq. (58) becomes

$$
\overline{b_{j}(\mathbf{y}, \mathbf{z} ; t)} \approx \frac{1}{N} \sum_{k=1}^{N} b_{j}\left(\mathbf{y}, \mathbf{z}^{k}\right) \quad \forall 1 \leqslant j \leqslant 2 m-2 l
$$

in which $\mathbf{z}^{k}$ is the $k$ th simulation of Eq. (41). For the deterministic approximation, Eq. (44) indicates that we need only solve for the deterministic evolution of the fast extents. We propose using Algorithm 2 to solve this partitioned reaction system, in which we choose to use only one simulation to evaluate Eq. (59) for the Langevin case. This Algorithm 2 is in fact identical to the one we previously proposed in Haseltine and Rawlings. ${ }^{17}$ The derivation presented in this paper, however, points out that using more than one simulation to evaluate Eq. (59) for the Langevin case is also possible. Such techniques are not considered in this paper, but are left for future investigations.

Our previous paper ${ }^{17}$ provides some heuristics for partitioning of the state $\mathbf{x}$ into fast and slow extents. We refer the interested reader to several more recent papers that address this issue in greater detail. ${ }^{30-33}$

ALGORITHM 2. Exact solution of the partitioned stochastic system for the Langevin and deterministic approximations.

Off-line. Determine the criteria for when and how the set $\mathbf{x}$ of $2 m$ reaction extends should be partitioned. Determine the stoichiometric matrices of the form given in Eq. (1) and reaction propensity laws for the unpartitioned [the matrix $\boldsymbol{\nu}$ and the $a_{k}(\mathbf{x})$ 's] and partitioned cases [the matrix $\boldsymbol{\nu}^{y}$, the matrix $\boldsymbol{\nu}^{z}$, and the $b_{k}(\mathbf{y}, \mathbf{z})$ 's]. Also, determine the necessary Langevin or deterministic equations for the fast reactions in the partitioned case.

Initialize. Set the time $t$ equal to zero. Set the number of species $\mathbf{n}$ to $\mathbf{n}_{0}$.

1. If the partitioning criteria established off-line are met, go to step 5.

2. Calculate

(a) the reaction propensities, $r_{k}=a_{k}(\mathbf{x})$, and

(b) the total reaction propensity, $r_{\text {tot }}=\sum_{k=1}^{2 m} r_{k}$.

3. Select two random numbers $p_{1}, p_{2}$ from the uniform distribution $(0,1)$.

Choose $j$ such that

$$
\text { Let } \tau=-\log \left(p_{1}\right) / r_{\text {tot }} \text {. }
$$

$$
\sum_{k=1}^{j-1} r_{k}<p_{2} r_{\text {tot }} \leqslant \sum_{k=1}^{j} r_{k}
$$

4. Let $t \longleftarrow t+\tau$.

Let $\mathbf{n} \leftarrow \mathbf{n}+\boldsymbol{\nu}_{j}^{T}$, where $\boldsymbol{\nu}_{j}$ is the $j$ th row of $\boldsymbol{\nu}$.

Go to step 1.

5. For subset $\mathbf{y}$, calculate

(a) the reaction propensities, $r_{k}^{y}=b_{k}(\mathbf{y}, \mathbf{z})$, and

(b) the total reaction propensity, $r_{\text {tot }}^{y}=\sum_{k=1}^{2 m-2 l} r_{k}^{y}$.

6. Select two random numbers $p_{1}, p_{2}$ from the uniform distribution $(0,1)$.

7. Determine $\hat{\nu}_{\mathbf{z}}=\left(\boldsymbol{\nu}^{z}\right)^{T}[\mathbf{z}(t+\tau)-\mathbf{z}(t)]$ by integrating $r_{\text {tot }}^{y}(t)$ and the subset of fast reactions $\mathbf{z}$ until the following condition is met:

$$
\int_{t}^{t+\tau} r_{\text {tot }}^{y}\left(t^{\prime}\right) d t^{\prime}+\log \left(p_{1}\right)=0 \quad \text { such that } r_{\text {tot }}^{y}(t)=\sum_{k=1}^{2 m-2 l} b_{k}(\mathbf{y}, \mathbf{z} ; t) .
$$

8. Let $t \leftarrow t+\tau$.

Let $\mathbf{n} \leftarrow \mathbf{n}+\hat{\boldsymbol{v}}_{z}$.

9. Choose $j$ such that

$$
\sum_{k=1}^{j-1} r_{k}^{y}<p_{2} r_{\text {tot }}^{y}(t) \leqslant \sum_{k=1}^{j} r_{k}^{y} .
$$

Current values of the $r_{k}^{y}$,s and $r_{\text {tot }}^{y}$ should be available from step 7.

10. Let $\mathbf{n} \leftarrow \mathbf{n}+\left(\boldsymbol{\nu}_{j}^{y}\right)^{T}$, where $\boldsymbol{\nu}_{j}^{y}$ is the $j$ th row of $\boldsymbol{\nu}^{y}$. Go to step 1 . 
TABLE I. Model parameters and reaction propensities for the enzyme kinetics example.

\begin{tabular}{ccr}
\hline \hline Parameter & Symbol & Value \\
\hline Forward reaction propensity (60a) & $a_{1}^{f}(\mathbf{x})$ & $k_{1}^{f} n_{E} n_{S}$ \\
Reverse reaction propensity (60a) & $a_{1}^{r}(\mathbf{x})$ & $k_{1}^{r} n_{E S}$ \\
Reaction propensity (60b) & $a_{2}^{f}(\mathbf{x})$ & $k_{2} n_{E S}$ \\
Reaction (60a) forward rate constant & $k_{1}^{f}$ & 20 \\
Reaction (60a) reverse rate constant & $k_{1}^{r}$ & 200 \\
Reaction (60b) rate constant & $k_{2}$ & 1 \\
Initial number of $E$ molecules & $n_{E_{0}}$ & 20 \\
Initial number of $S$ molecules & $n_{S_{0}}$ & 10 \\
Initial number of $E S$ molecules & $n_{E S_{0}}$ & 0 \\
Initial number of $P$ molecules & $n_{P_{0}}$ & 0 \\
\hline \hline
\end{tabular}

\section{EXAMPLES}

We now consider two motivating examples that illustrate both when the various approximations should (and should not) be used and the accuracy of the approximations. For clarity, we first briefly review the nomenclature that indicates which approximations, if any, are performed in a given simulation. We can either perform a purely stochastic simulation on the unpartitioned reaction system or we can partition the system into fast and slow reactions. For this partitioned case, a stochastic-equilibrium simulation equilibrates the fast reactions, a stochastic-Langevin simulation treats the fast reactions as Langevin equations, and a stochastic-deterministic simulation treats the fast reactions deterministically.

\section{A. Enzyme kinetics}

We consider the simple enzyme kinetics problem

$$
\begin{gathered}
E+\underset{k_{1}^{r}}{\stackrel{k_{1}^{f}}{\rightleftharpoons}} E S \quad \epsilon_{1}, \\
\stackrel{k_{2}}{\longrightarrow} E+P \quad \epsilon_{2} .
\end{gathered}
$$

The model parameters and the reaction propensities are given in Table I. For this example, the first forward and reverse reactions occur many times before the expected time of one second reaction. Hence we partition the reaction extents as follows: $\epsilon_{2}$ comprises the subset of slow reactions $\mathbf{y}$ and $\epsilon_{1}$ comprises the subset of fast reactions $\mathbf{z}$.

For this example, the hybrid simulation techniques (i.e., approximating the fast reactions using deterministic or Langevin equations) are clearly not valid. Changes in the fast reactions lead to large changes in the fast-reaction propensities due to the small number of molecules involved. Rather, the fast reactions equilibrate (relax) significantly faster than the slow reaction. Therefore, we consider using the equilibrium approximation to address this example.

We calculate the averages of all species using 1000 simulations sampled at a time interval of 0.1 units. We use both the stochastic-equilibrium and exact simulations to compute these averages. Figure 1 presents the results of the comparison. The stochastic-equilibrium simulation provides an excellent reconstruction of the mean behavior. The exact

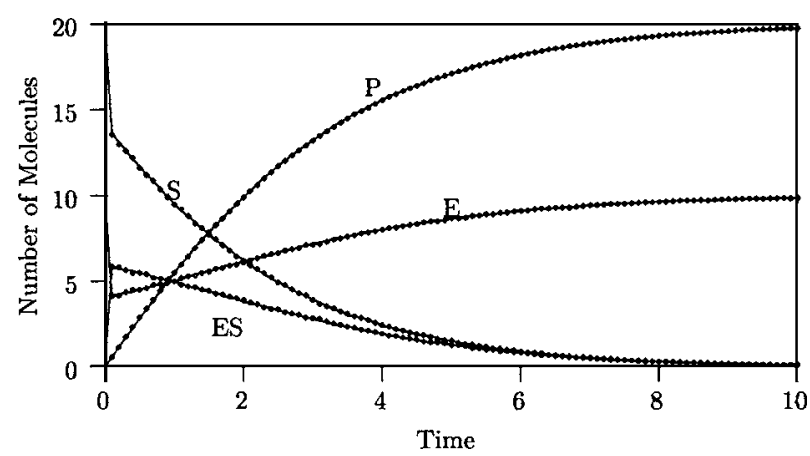

FIG. 1. Comparison of the stochastic-equilibrium simulation (points) to exact stochastic simulation (solid lines) based on 1000 simulations.

simulation requires roughly 27 times the amount of computational expense as the stochastic-equilibrium simulation.

We refer the interested reader to Cao et al. ${ }^{16}$ for additional examples and discussion of the equilibrium approximation. While their derivation of the equilibrium approximation differs from ours, their simulation algorithm is similar to our Algorithm 1.

\section{B. Simple crystallization}

We consider a simplified reaction system for the crystallization of species $A$ presented previously by Haseltine and Rawlings: ${ }^{17}$

$$
\begin{aligned}
& 2 A \stackrel{k_{1}}{\rightarrow} B \quad \epsilon_{1}, \\
& A+C \stackrel{k_{2}}{\rightarrow} D \quad \epsilon_{2} .
\end{aligned}
$$

The model parameters and the reaction propensities are given in Table II. For this example, the first reaction occurs many more times than the second reaction. Hence we partition the extents of reaction $\left(\epsilon_{i}{ }^{\prime}\right.$ s) as follows: ${ }^{34} \epsilon_{2}$ comprises the subset of slow reactions $\mathbf{y}$ and $\epsilon_{1}$ comprises the subset of fast reactions $\mathbf{z}$.

For this example, the equilibrium approximation is clearly not valid. Equilibrating the fast reaction would result in all of the $A$ forming $B$, and hence no $C$ would ever react. Rather, the relaxation times of the fast and slow reactions are comparable, so we should approximate the fast reactions appropriately. Here, we approximate the fast-reaction subset using the Langevin approximation (stochastic-Langevin simulation) and attempt to reconstruct the first two moments

TABLE II. Model parameters and reaction propensities for the simple crystallization example.

\begin{tabular}{ccr}
\hline \hline Parameter & Symbol & Value \\
\hline Reaction propensity (61a) & $a_{1}(\mathbf{x})$ & $\frac{1}{2} k_{1} n_{A}\left(n_{A}-1\right)$ \\
Reaction propensity (61b) & $a_{2}(\mathbf{x})$ & $k_{2} n_{A} n_{C}$ \\
Reaction (61a) rate constant & $k_{1}$ & $1 \times 10^{-7}$ \\
Reaction (61b) rate constant & $k_{2}$ & $1 \times 10^{-7}$ \\
Initial number of $A$ molecules & $n_{A_{0}}$ & $1 \times 10^{6}$ \\
Initial number of $B$ molecules & $n_{B_{0}}$ & 0 \\
Initial number of $C$ molecules & $n_{C_{0}}$ & 10 \\
Initial number of $D$ molecules & $n_{D_{0}}$ & 0 \\
\hline \hline
\end{tabular}


of each species. The Langevin equations are integrated using the Euler-Murayama method ${ }^{35}$ with a time increment of 0.01 . We account for the time-varying propensity of the slow reaction exactly as outlined by Haseltine and Rawlings. ${ }^{17}$ Figure 2 compares these results to the exact stochastic results for 10000 simulations. The approximation accurately reconstructs the mean and standard deviation for all species with a 13-fold reduction in computational expense. We refer the interested reader to Haseltine and Rawlings ${ }^{17}$ for results in which the fast reaction is approximated deterministically (stochastic-deterministic simulation). This approximation accurately reconstructs all of the means as well as the standard deviations for species $C$ and $D$, but fails to reconstruct the standard deviations for species $A$ and $B$. This phenomenon is expected because approximating $\epsilon_{1}$ deterministically neglects all fluctuations caused by the first reaction. However, the stochastic-deterministic simulation has the additional benefit of providing a bound on the computational expense. For example, any increase in the initial number of $A$ molecules $n_{A_{0}}$ increases the computational expense of exact stochastic simulation, whereas the computational expense of the stochastic-deterministic simulation remains constant regardless of the value of $n_{A_{0}}$.

\section{DISCUSSION AND CONCLUSIONS}

The primary contribution of this work is the development of a conceptual framework that gives rise to both the hybrid and equilibrium approximations for stochastic chemical kinetics. This task is facilitated by partitioning a purely stochastic reaction system using net reaction extents into subsets of slow and fast reactions. Using order-of-magnitude arguments, we derive approximate Markov evolution equations for the slow-extent marginal and the fast extents conditioned on the slow extents. The evolution equation for the fast extents conditioned on the slow extents is a closed-form expression, whereas the evolution equation for the slowextent marginal depends on this conditional probability. Using relaxation-time arguments, we can propose two approximations for the fast extents: a Langevin or deterministic approximation when both fast and slow extents exhibit similar relaxation times and an equilibrium approximation when the fast extents relax faster than the slow extents. Derivation of the Langevin and deterministic approximations points out several weaknesses in our previous justification of the hybrid system. ${ }^{17}$ Many of these weaknesses were also noted independently by Goutsias. ${ }^{19}$ The equilibrium assumption is similar in nature to the slow-reaction simulation recently proposed in the literature by Cao et al. ${ }^{16}$ These authors did an excellent job of deducing how an approximate simulation should behave when a subset of the total reactions equilibrate stochastically. However, their approach partitions chemical species as opposed to reactions. This choice obscures the fact that reactions are equilibrating, not chemical species. Additionally, the choice of introducing a virtual fast system leaves open the question of how their slow-scale stochastic simulation and the original master equation are connected. We believe that our approach (1) more clearly points out what terms are neglected in the derivation of the equilibrium ap-
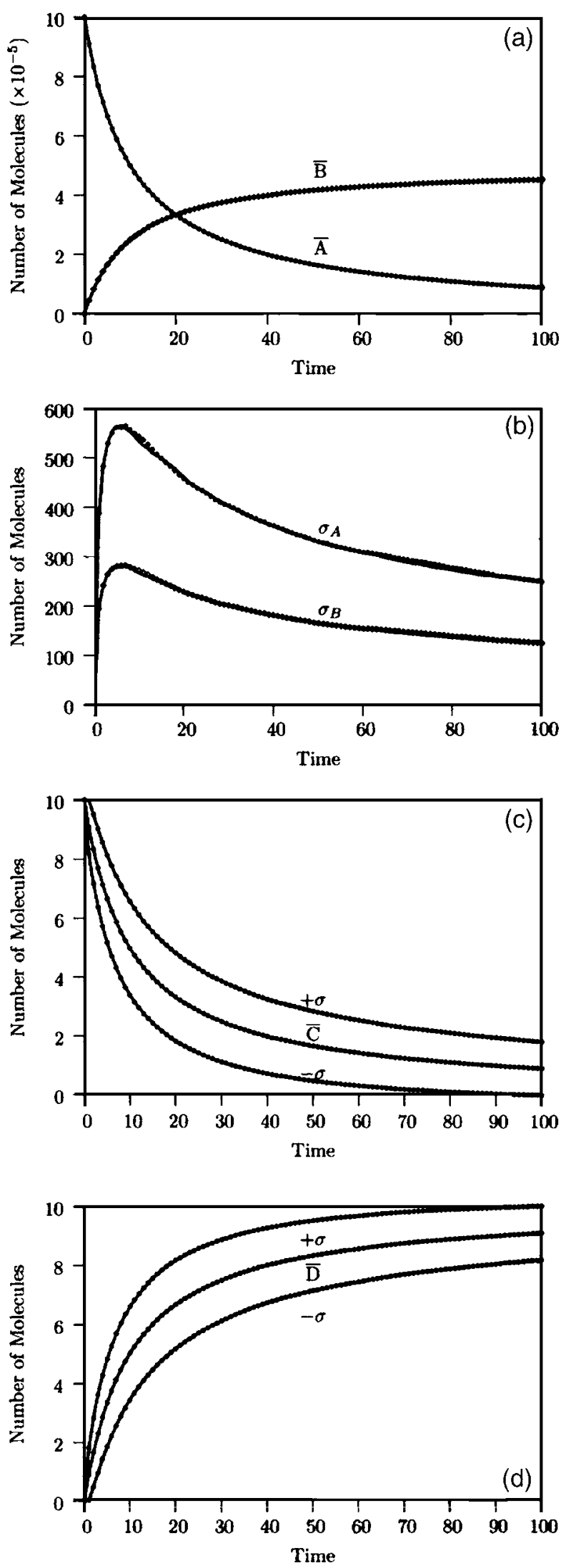

FIG. 2. Comparison of exact stochastic-Langevin simulation (points) to exact stochastic simulation (lines) based on 10000 simulations and a Langevin time step of 0.01. (a) Comparison of the means $\bar{A}$ and $\bar{B}$ for species $A$ and $B$, respectively. (b) Comparison of the standard deviations $\sigma_{A}$ and $\sigma_{B}$ for species $A$ and $B$, respectively. (c) Comparison of the mean $(\bar{C})$ and standard deviation $( \pm \sigma)$ for species $C$. (d) Comparison of the mean $(\bar{D})$ and standard deviation $( \pm \sigma)$ for species $D$. 
proximation and (2) provides a tighter connection between the original and approximate master equations. Also, the approximate, coupled master equations (27) are similar to the ones independently proposed by both Goutsias ${ }^{19}$ and Haseltine. ${ }^{18}$ However, our derivation differs in the subtle choice of representing the system using net extents as opposed to irreversible extents. As noted previously, under standard assumptions such as conservation of elements, a finite number of reactions, and finite stoichiometries for reaction events, the net extent representation yields a master equation with a finite number of entries, whereas the irreversible extent representation yields an infinite number of entries, which makes analysis of the approximate master equation more difficult. For example, given the standard assumptions enumerated above, it is possible to prove that the approximate marginal density $P_{A}(\mathbf{y} ; t)>0 \forall t_{0}<t<\infty$ because there are only a finite number of states in the joint density $P_{A}(\mathbf{y}, \mathbf{z} ; t)$. For the infinite dimensional case, it is not clear if there exist some $\mathbf{y}$ for which $P_{A}(\mathbf{y} ; t)=0$; therefore, the question arises as to whether or not $P_{A}(\mathbf{z} \mid \mathbf{y} ; t)$ is well defined. Additionally, the irreversible extent representation renders a master equation that may never equilibrate to a steady state. In the specific case of all reversible reactions, each irreversible extent tends to infinity in the limit as $t \rightarrow \infty$. It is only in the net extent or species number coordinates that a true steady state can be realized. This issue is tacitly present but never addressed by Goutsias ${ }^{19}$ when the stochastic quasiequilibrium assumption is introduced. Goutsias ${ }^{19}$ proposes that some fast-reaction propensities should be approximately equal, i.e.,

$$
c_{j}^{f}(\mathbf{y}, \mathbf{z} ; t) \approx c_{j}^{r}(\mathbf{y}, \mathbf{z} ; t) .
$$

This statement is misleading because Eq. (62) is never exactly true in any limit, except for some fortuitous choice of rate constants. On the other hand, equilibration of the conditional probability distribution as given by Eq. (31b) is true in the limit as $t \rightarrow \infty$. Finally, we note that each of the examples presented by Goutsias ${ }^{19}$ employs strategies for evaluating linear combinations of the irreversible extents that correspond exactly to the net extent.

Neither Cao et al. ${ }^{16}$ nor Goutsias ${ }^{19}$ address the case in which the fast- and slow-reaction extents exhibit similar relaxation times. The simple crystallization example presented in this paper illustrates an example of this case. In this example, exact stochastic simulation is computationally expensive because the fast reaction fires substantially more times than the slow reaction. However, the fast and slow reactions are relaxing at similar rates. Applying the equilibrium approximation to this example would yield the incorrect conclusion that the slow reaction never occurs. It is precisely this case that our previous paper ${ }^{17}$ addressed.

By equilibrating the fast-reaction subset, we can substantially reduce the computational requirement by integrating the system over a much larger time step than the exact stochastic simulation. This method requires solving for the equilibrium distribution of the fast reactions. If there are few fast extents or many of the fast extents are independent of one another, then exactly solving for this distribution is possible as illustrated by the enzyme kinetics example. If there are a large number of coupled fast extents, then exact solution may not be computationally feasible. For example, consider the coupled, fast reactions

$$
A+E \rightleftharpoons B+E \rightleftharpoons C+E \rightleftharpoons D+E .
$$

A minimal representation of these reactions requires three (reversible) extents of reaction. The resulting system is difficult to solve given a reasonable number of molecules for each species. Here, simulation methods may prove useful in approximating the equilibrium distributions.

By approximating the fast-reaction subset using Langevin equations, we can reduce the computational requirement by integrating the system over a much larger time step than the exact stochastic simulation. However, we must now employ schemes for integrating stochastic differential equations. By approximating the fast-reaction subset deterministically, we can bound the computational requirements for simulation of the system. For this case, we can employ existing and robust ordinary differential equation solvers for integration of this reaction subset. In contrast, the computational expense for exact stochastic simulation scales with the number of reaction events.

The partitioning techniques presented here sacrifice some numerical accuracy for a bound on the computational load. By equilibrating some fast reactions, one cannot expect to accurately reconstruct statistics for species affected by these fast reactions at fine time scales. However, we are often interested in the macroscopic behavior of the system, and it may not be possible to even observe a physical system at such fine time scales. Approximating some discrete, molecular reaction events as continuous events via the Langevin approximation loses the discrete nature of the entire system. However, as illustrated by the simple crystallization example, this approximation still accurately reconstructs at least the first two moments of each reacting species. Furthermore, approximating fast reactions deterministically eliminates all fluctuations contributed to the system by these reactions. Depending upon the system and the modeling objective, though, these sacrifices may be acceptable.

The approach described here represents only a small fraction of the approximations that should prove useful for simulating stochastic chemical kinetics. For example, Tian and Burrage ${ }^{8}$ propose classifying reactions into three categories (slow, intermediate, and fast), then making appropriate approximations for each category (exact simulation for slow reactions, $\tau$ or binomial-leaping for intermediate reactions, and Langevin or deterministic simulation for fast reactions). The techniques presented in this paper could easily be extended to derive appropriate master equations for such cases. Also, we did not present algorithms for other possible simulation methods such as the next- or first-reaction methods. These extensions follow directly from the joint probability function $P(\tau, \mu)$ given by Eq. (47). However, a recent numerical study by Cao et al. ${ }^{4}$ shows that these methods are 
often less efficient than the direct method for exact simulation. Additionally, we expect the majority of the reduction in computational expense to result from the fast-reaction approximations.

Finally, we attempt to classify each of the recently proposed simulation algorithms. We note that the computational burden of stochastic simulation stems from three primary phenomena:

1. Some subset of reversible reactions occur frequently, whereas the remaining reactions occur rarely.

2. Some subset of irreversible reactions occur frequently, ${ }^{36}$ whereas the remaining reactions occur rarely.

3. Some subset of the chemical species react so quickly that their average number is significantly less than one throughout the course of the simulation.

The first phenomenon is attributable to stiffness in the underlying master equation for these system. For deterministic kinetics, such stiffness can be handled by employing an equilibrium approximation. This paper, Goutsias, ${ }^{19}$ and Cao et al. ${ }^{16}$ each consider simulation algorithms for the stochastic equivalent of the equilibrium approximation. The second phenomenon is unique to the discrete stochastic formulation of chemical kinetics and arises due to the fact that exact simulation methods can advance only one reaction at a time. Only our previous paper ${ }^{17}$ and this paper address this phenomenon. None of the above-mentioned articles consider the third phenomenon. In a deterministic setting, this phenomenon is typically addressed by applying the quasi-steadystate assumption (QSSA) to equilibrate the rate of change for a given chemical species. In terms of our previous example, reaction (28), we could choose species $B$ as the quasi-steadystate species and set $a_{1}^{f}(\mathbf{x})-a_{1}^{r}(\mathbf{x})-a_{2}^{f}(\mathbf{x})=0$. For the discrete master equation, however, it is unlikely that such a situation can arise due to the integer nature of all chemical species. The most likely situation is for either $a_{1}^{f}(\mathbf{x})>0$ and $a_{1}^{r}(\mathbf{x})$ $=a_{2}^{f}(\mathbf{x})=0\left(\right.$ for $\left.n_{B}=0\right)$ or $a_{1}^{r}(\mathbf{x}), a_{2}^{f}(\mathbf{x}) \gg a_{1}^{f}(\mathbf{x})\left(\right.$ for $\left.n_{B}>0\right)$. In this case, we would expect to almost never find a $B$ molecule in an exact simulation. Although Rao and Arkin ${ }^{15}$ recently addressed this issue, they assumed a Markovian form for their governing master equation rather than derive it directly from the original master equation (2). A tighter connection between the original and approximate systems should be possible.

\section{ACKNOWLEDGMENTS}

One of the authors (E.L.H.) was supported by an NLM training grant to the Computation and Informatics in Biology and Medicine Training Program (NLM 5T15LM007359). We gratefully acknowledge the financial support of the industrial members of the Texas-Wisconsin Modeling and ControlConsortium. All simulations were performed using OCTAVE (http://www.octave.org). OCTAVE is freely distributed under the terms of the GNU General Public License.

\section{APPENDIX: NOTATION}

$a_{j}^{f}(\mathbf{n})=j$ th forward reaction propensity (rate)

$a_{j}^{r}(\mathbf{n})=j$ th reverse reaction propensity (rate)

$\overline{b_{j}(\mathbf{y}, \mathbf{z})}=j$ th slow-reaction rate averaged over values of the fast extents

$b_{j}^{f}(\mathbf{y}, \mathbf{z})=j$ th forward slow-reaction rate

$b_{j}^{r}(\mathbf{y}, \mathbf{z})=j$ th reverse slow-reaction rate

$C=$ matrix of reaction propensities

$c_{j}^{f}(\mathbf{y}, \mathbf{z})=j$ th forward fast-reaction rate

$c_{j}^{r}(\mathbf{y}, \mathbf{z})=j$ th reverse fast-reaction rate

$\boldsymbol{I}=$ identity matrix

$k_{j}=$ rate constant for reaction $k$

$N=$ number of Monte Carlo simulations

$\mathbb{N}_{p}^{f}=$ set containing the molecule space for the fast partitioned species

$\mathbb{N}_{p}^{s}=$ set containing the molecule space for the slow partitioned species

$n_{j}=$ number of molecules for species $j$

$n_{j o}=$ initial number of molecules for species $j$

$\mathbf{n}=$ number of molecules for all reaction species

$\mathbf{n}^{f}=$ number of molecules for the fast-reaction species

$\mathbf{n}^{s}=$ number of molecules for the slow-reaction species

$\mathbf{n}_{0}=$ initial number of molecules for all reaction species

$\mathbf{n}_{0, j}=j$ th initial number of molecules for all reaction species

$\mathbf{p}=$ probability vector for all possible values of the extents of reaction

$P=$ probability

$P_{A}=$ approximate probability (reduced by orderof-magnitude arguments)

$p=$ random number from the uniform distribution $(0,1)$

$r_{\text {tot }}=$ sum of reaction rates

$r_{\text {tot }}^{y}=$ sum of reaction rates for the slow-reaction partition

$t=$ time

$t_{0}=$ initial time

$\boldsymbol{W}=$ vector of Wiener processes

$X=$ set containing the total accessible extent space

$X_{p}=$ set containing the extent space for the partitioned reactions

$\mathbf{x}=$ state of the system in terms of extents

$\mathrm{Y}=$ set containing the total accessible slowextent space

$\mathbb{Y}_{p}=$ set containing the slow-extent space for the partitioned reactions

$\mathbf{y}=$ subset of slow-reaction extents

$\mathbf{z}=$ subset of fast-reaction extents

$\mathrm{Z}=$ set containing the total accessible fast-extent space

$Z_{p}=$ set containing the fast-extent space for the partitioned reactions

$\boldsymbol{Z}=$ subset of fast-reaction extents scaled by $\Omega$

$\epsilon=$ extent of reaction 
$\mu=$ one possible reaction in the stochastic kinetics framework

$\boldsymbol{\nu}=$ stoichiometric matrix

$\sigma=$ standard deviation

$\tau=$ time of the next stochastic reaction

$\Omega=$ characteristic system size

${ }^{1}$ D. T. Gillespie, J. Comput. Phys. 22, 403 (1976).

${ }^{2}$ D. T. Gillespie, J. Phys. Chem. 81, 2340 (1977).

${ }^{3}$ M. A. Gibson and J. Bruck, J. Phys. Chem. A 104, 1876 (2000).

${ }^{4}$ Y. Cao, H. Li, and L. R. Petzold, J. Chem. Phys. 121, 4059 (2004).

${ }^{5}$ D. T. Gillespie, J. Chem. Phys. 115, 1716 (2001).

${ }^{6}$ D. T. Gillespie and L. R. Petzold, J. Chem. Phys. 119, 8229 (2003).

${ }^{7}$ M. Rathinam, L. R. Petzold, Y. Cao, and D. T. Gillespie, J. Chem. Phys. 119, 12784 (2003).

${ }^{8}$ T. Tian and K. Burrage, J. Chem. Phys. 121, 10356 (2004).

${ }^{9}$ A. Chatterjee, D. G. Vlachos, and M. A. Katsoulakis, J. Chem. Phys. 122, 024112 (2005).

${ }^{10}$ J. A. M. Janssen, J. Stat. Phys. 57, 171 (1989).

${ }^{11}$ J. A. M. Janssen, J. Stat. Phys. 57, 187 (1989).

${ }^{12}$ M. O. Vlad and A. Pop, Physica A 155, 276 (1989).

${ }^{13}$ H. Haken, Synergetics: An Introduction: Nonequilibrium Phase Transitions and Self-organization in Physics, Chemistry, and Biology, 3rd ed. (Springer, New York, 1983).

${ }^{14}$ N. G. van Kampen, Phys. Rep. 124, 69 (1985).

${ }^{15}$ C. V. Rao and A. P. Arkin, J. Chem. Phys. 118, 4999 (2003).

${ }^{16}$ Y. Cao, D. T. Gillespie, and L. R. Petzold, J. Chem. Phys. 122, 014116 (2005).
${ }^{17}$ E. L. Haseltine and J. B. Rawlings, J. Chem. Phys. 117, 6903 (2002).

${ }^{18}$ E. L. Haseltine, Ph.D. thesis, University of Wisconsin-Madison, 2005.

${ }^{19}$ J. Goutsias, J. Chem. Phys. 122, 184102 (2005).

${ }^{20}$ Goutsias (Ref. 19) uses the terminology "degree of advancement" as opposed to irreversible extent.

${ }^{21}$ M. O. Vlad and J. Ross, J. Phys. Chem. B 101, 8756 (1997).

${ }^{22}$ M. O. Vlad and J. Ross, J. Phys. Chem. A 104, 3159 (2000).

${ }^{23}$ K. Ishida, Phys. Chem. Chem. Phys. 2, 4165 (2000).

${ }^{24}$ D. T. Gillespie, Physica A 188, 404 (1992).

${ }^{25}$ C. W. Gardiner, Handbook of Stochastic Methods for Physics, Chemistry, and the Natural Sciences, 2nd ed. (Springer, Berlin, Germany, 1990).

${ }^{26}$ D. T. Gillespie, J. Chem. Phys. 113, 297 (2000).

${ }^{27}$ T. G. Kurtz, J. Chem. Phys. 57, 2976 (1972).

${ }^{28}$ D. T. Gillespie, Markov Processes: An Introduction for Physical Scientists (Academic, New York, 1992).

${ }^{29}$ H. A. van der Vorst, in Iterative Krylov Methods for Large Linear Systems, Cambridge Monographs on Applied and Computational Mathematics No. 13 (Cambridge University Press, New York, NY, 2003).

${ }^{30}$ J. Puchalka and A. M. Kierzek, Biophys. J. 86, 1357 (2004).

${ }^{31}$ K. Vasudeva and U. S. Bhalla, Bioinformatics 20, 78 (2004).

${ }^{32}$ K. Burrage, T. Tian, and P. Burrage, Prog. Biophys. Mol. Biol. 85, 217 (2004).

${ }^{33}$ H. Salis and Y. Kaznessis, J. Chem. Phys. 122, 054103 (2005).

${ }^{34}$ Reactions are partitioned on the basis of the magnitude of their reaction propensities, not their rate constants.

${ }^{35}$ T. C. Gard, Introduction to Stochastic Differential Equations (Marcel Dekker, New York, 1988).

${ }^{36}$ This case includes reversible reactions in which either the forward or reverse reaction dominates. 